\title{
Heterogeneous interactions promote crystallization and spontaneous resolution of chiral molecules
}

\author{
Supporting Information \\ John E. Carpenter and Michael Grünwald \\ Department of Chemistry, University of Utah, Salt Lake City, 84112 Utah, USA.
}

\section{Contents:}

Part 1. MD Simulations

1.1 Crystallization Protocol

1.2 Reproducibility of Crystallization Results

1.3 Results with Uniform Interactions $\left(\varepsilon_{S}=1 \varepsilon\right)$

1.4 Low Density Crystals at $\varepsilon_{S}>7$

1.5 Enantiopure Propensity of Oligomers

1.6 Charge-like Interactions

\section{Part 2. POLYNUM}

2.1 Algorithm Overview

2.2 Unit Cell Determination

2.3 Constructing an Incidence Matrix

2.4 Removing Equivalent Solutions

2.4.1 Contact Vectors

2.4.2 Unique Tags

2.5 Examples of Polymorph Solutions

2.6 Exponential Increase in Polymorph Solutions with Added Holes

2.7 Verification of Crystallinity Algorithm and Polymorph Enumeration Procedure

2.8 Increase in Energy of Polymorphs as a Function of Packing Fraction

Part 3. Determining Crystal Quality (CQ)

\subsection{Validation}

3.2 Examples

Part 4. Free Energy Calculations

4.1 Procedure

4.2 Results

Part 5. Competing Polymorphs

5.1 Effects of Orientational Constraints on Crystallization and Separation Propensity

5.2 Enantiomer Ratios vs. Binomial Statistics

Part 6. Predicting Crystallization Using Machine Learning Techniques

6.1 Training a NN to Learn $\Delta E$ for Fixed Molecular Shape

6.2 NN Predicts Molecules that Form Enantiopure Crystals in Simulations

6.3 Clustering of Interaction Vectors

6.4 Training a NN to Predict $\triangle E$ Based on Descriptors of Molecular Shape

\section{References}




\section{Part 1. MD Simulations}

1.1 Crystallization Protocol: MD simulation are carried out in the NVT ensemble using Langevin dynamics and are initiated from dispersed configurations of 5184 molecules at a density of 0.04 molecules/ $\sigma^{2}$.

- Stage I. Simulations start at a temperature of $T=1.3 \frac{\epsilon}{k_{B}}$, which is high enough to ensure an initial metastable equilibrium of monomers and small oligomers. The temperature is then lowered rapidly, at a rate of $3.0 \frac{\epsilon}{k_{B}}$ per $10^{8}$ timesteps, until a cluster of 50 molecules or larger is detected in the simulation cell. A cluster is defined by a simple nearest neighbor criterion: Functional groups on different molecules are considered to be bonded if their distance is smaller than $1.1 \sigma$. All pairs of molecules that have at least one bond between their functional groups are considered part of the same cluster. Once the first cluster of 50 molecules is identified, the configuration of the system is saved.

- Stage II. Starting from the last configuration obtained in stage I, several trajectories of $10^{6}$ time steps each are advanced from this initial configuration at different temperatures, until a temperature $T_{g}$ is identified at which the largest cluster grows at a rate of $x_{g}=5 \pm 1$ molecules per $10^{6}$ time steps. The system is then simulated at that temperature until the largest cluster has reached a size of 100 molecules. At this point, the procedure is repeated, i.e., several temperature levels are scanned and a (possibly) new temperature $T_{g}$ is selected that enables continued growth at the same rate $x_{g}$. The simulation is then continued at $T_{g}$ until the largest cluster has reached a size of 200 molecules. The purpose of this stage is to enable growth of clusters with at least partial order. The rate $x_{g}$ was chosen as a compromise between slow growth conducive to ordered assembly and computational speed.

- Stage III. In the final stage of the protocol, the temperature is slowly increased at a rate of $0.2 \frac{\epsilon}{k_{B}}$ over the course of $10^{8}$ time steps. Every $10^{6}$ time steps, the largest 5 clusters in the simulation cell are identified and each cluster is classified broadly as either enantiopure (if its enantiomeric composition is 4:1 or larger) or racemic. Based on this categorization, CQ scores are determined for each cluster and recorded. The largest CQ scores achieved by any cluster during this stage of the protocol is then recorded as the overall CQ score of the simulation, as reported throughout the main text. Clusters with maximum CQ values for $\epsilon_{\mathrm{s}}=5 \epsilon$ are shown in Fig. 2 of the main text.

1.2 Reproducibility of Crystallization Results: Crystallization outcomes reported in the main manuscript were obtained from single simulation runs using the simulation protocol described above. To gauge the sensitivity of simulation outcomes to changes in initial conditions, we have performed two independent simulation runs for a few systems. The results are reported in Table $\mathrm{S} 1$. We have furthermore confirmed that crystallization outcomes are not sensitive to moderate changes in molecular concentration, as shown in Table S2. 


\begin{tabular}{|c|c|c|c|c|}
\hline Shape & Interaction & Rac/Pure & $\boldsymbol{C Q ~ S i m u l a t i o n ~ 1 ~}$ & $\boldsymbol{C Q}$ Simulation 2 \\
\hline \multirow{2}{*}{1} & \multirow{2}{*}{$3: 5$} & Rac & 0.60 & 0.64 \\
\cline { 3 - 5 } & \multirow{2}{*}{8} & Pure & 0 & 0 \\
\hline \multirow{2}{*}{8} & Rac & 0.14 & 0.11 \\
\cline { 3 - 5 } & & Pure & 0.64 & 0.63 \\
\hline \multirow{2}{*}{8} & \multirow{2}{*}{$3: 5$} & Rac & 0.65 & 0.60 \\
\cline { 3 - 5 } & & Pure & 0 & 0 \\
\hline \multirow{2}{*}{10} & \multirow{2}{*}{$1: 4$} & Rac & 0.74 & 0.73 \\
\cline { 3 - 5 } & & Pure & 0 & 0 \\
\hline
\end{tabular}

Table S1: Largest $\mathrm{CQ}$ values of racemic and enantiopure clusters obtained in two independent simulations of 4 different racemic solutions. In all cases, $\epsilon_{\mathrm{s}}=5 \epsilon$.

\begin{tabular}{|c|c|c|c|}
\hline Interactions & $\begin{array}{c}\mathbf{C Q}_{\mathbf{1}} \\
\boldsymbol{\rho}=\mathbf{0 . 0 4 \boldsymbol { \sigma } ^ { - 2 }}\end{array}$ & $\begin{array}{c}\boldsymbol{C Q}_{2} \\
\boldsymbol{\rho}=\mathbf{0 . 0 1} \boldsymbol{\sigma}^{-2}\end{array}$ & $\boldsymbol{C Q}_{\mathbf{1}}-\boldsymbol{C Q}_{\mathbf{2}}$ \\
\hline $1: 1$ & -0.58 & -0.46 & -0.12 \\
$2: 2$ & -0.69 & -0.73 & 0.04 \\
$3: 3$ & -0.55 & -0.57 & 0.02 \\
$4: 4$ & -0.34 & -0.37 & 0.03 \\
$5: 5$ & -0.47 & -0.55 & 0.08 \\
$1: 2$ & -0.50 & -0.49 & -0.01 \\
$1: 3$ & -0.73 & -0.75 & 0.03 \\
$1: 5$ & -0.50 & -0.46 & -0.03 \\
$2: 3$ & -0.86 & -0.78 & -0.08 \\
$2: 4$ & -0.38 & -0.28 & -0.10 \\
$2: 5$ & -0.58 & -0.21 & -0.37 \\
$3: 4$ & -0.72 & -0.52 & -0.20 \\
$3: 5$ & -0.71 & -0.72 & 0.01 \\
$4: 5$ & -0.73 & -0.70 & -0.04 \\
\hline Average & -0.60 & -0.54 & -0.05 \\
\end{tabular}

Table S2: Largest CQ values obtained in simulations of enantiopure solutions of shape s2 at different concentrations. In all cases, $\epsilon_{\mathrm{s}}=5 \epsilon$. Crystallization outcomes reported in the main text were obtained at $\rho=0.04 \sigma^{-2}$. 


\subsection{Results with Uniform Interactions $\left(\varepsilon_{s}=1 \varepsilon\right)$}

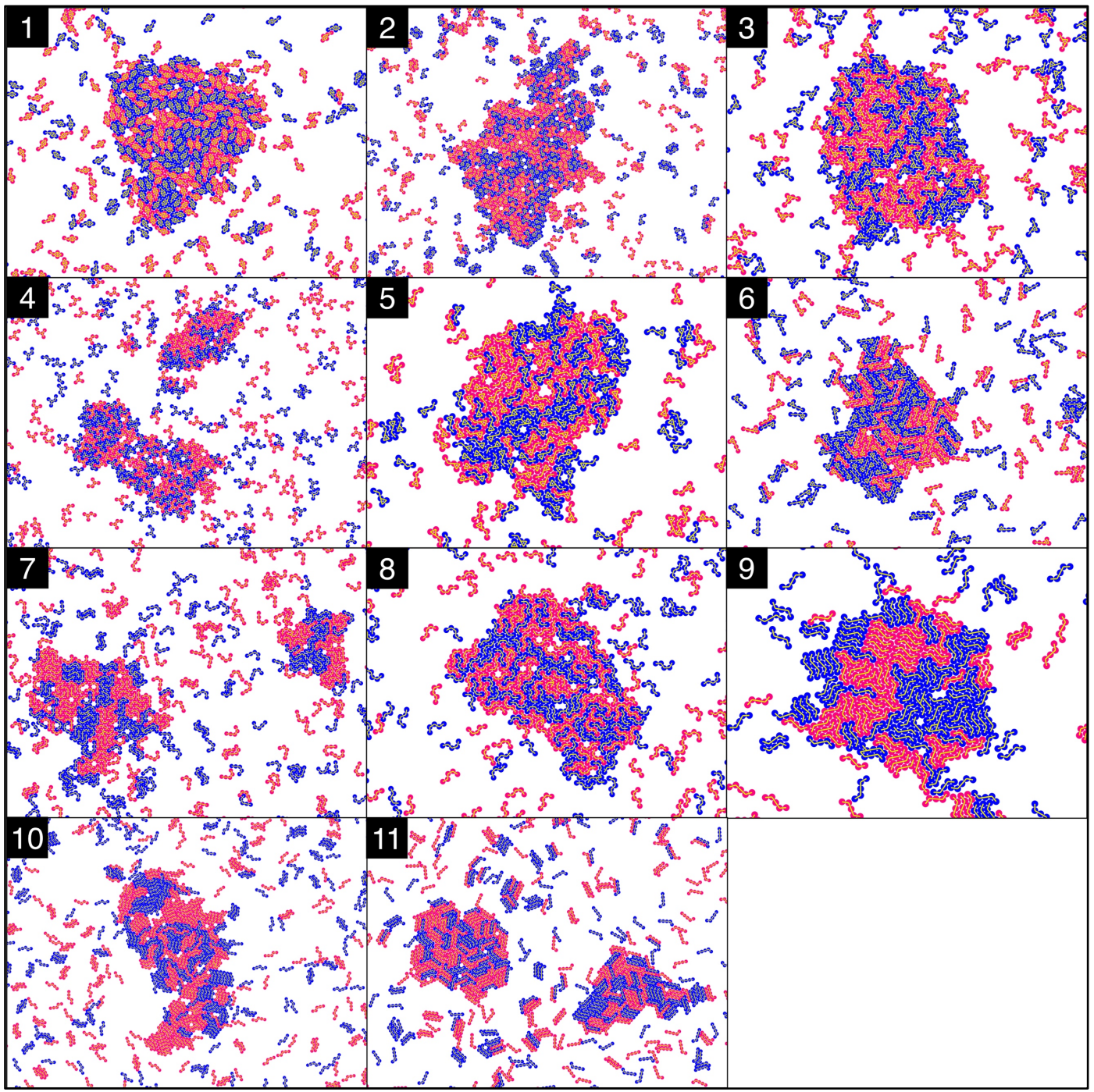

Figure S1: Snapshots of molecular clusters appearing in MD simulations of racemic solutions with uniform interactions across functional groups, $\epsilon_{s}=\epsilon$. Different panels show results for shapes s1-s11. No crystals were observed. 


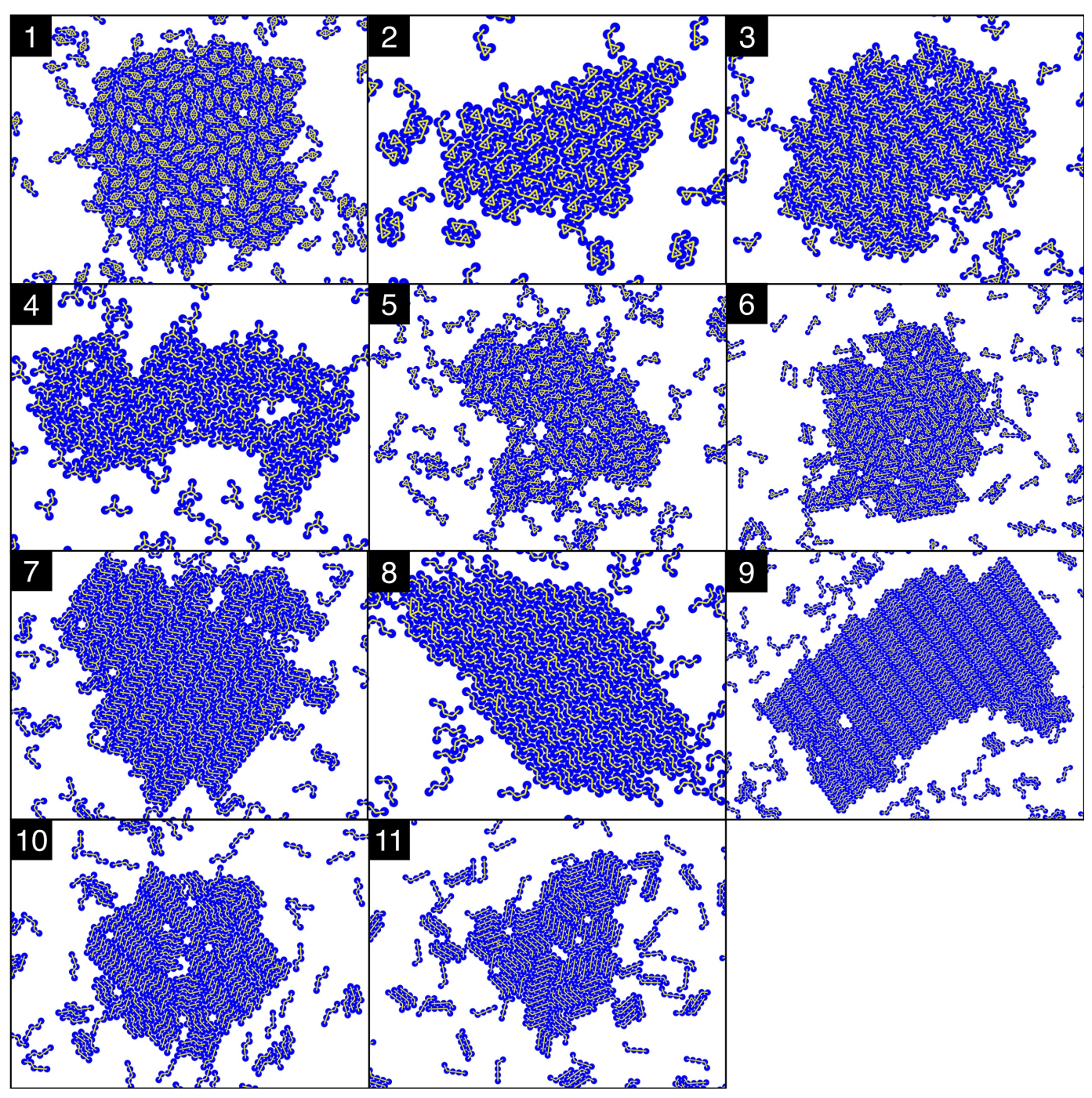

Figure S2: Snapshots of molecular clusters appearing in MD simulations of pure solutions with uniform interactions across functional groups, $\epsilon_{s}=\epsilon$. Different panels show results for shape s1-s11. Shapes s7, s8, and s9 formed crystals. 


\subsection{Low Density Crystals at $\varepsilon_{s}>7$ :}

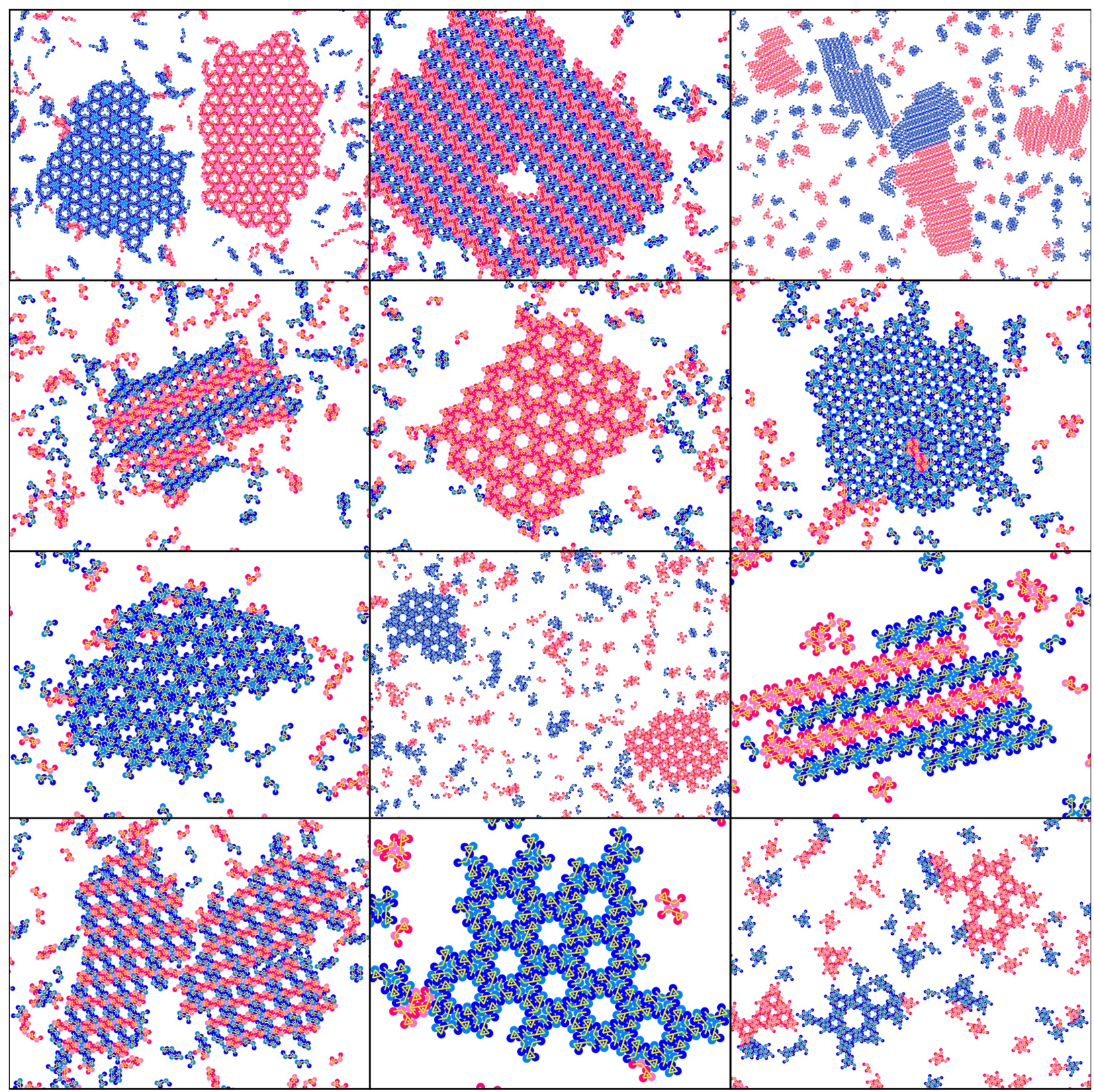

Figure S3: Snapshots of molecular clusters appearing in MD simulations of racemic solutions with randomly selected interaction vectors with strongly heterogeneous interactions $\left(47^{\circ}<\varphi_{\vec{\epsilon}}<60^{\circ}\right)$. 
1.5 Enantiopure Propensity of Oligomers: We have observed that the majority of oligomers present in our MD simulations at high temperatures are enantiopure. We show this in Figure S4, by comparing the number of like-contacts (enantiopure oligomers) to non-like-contacts (racemic oligomers), in MD simulations where the temperature is too high for structure formation. As can be see, in a majority of these simulations, like-contacts (enantiopure oligomers) are favored.

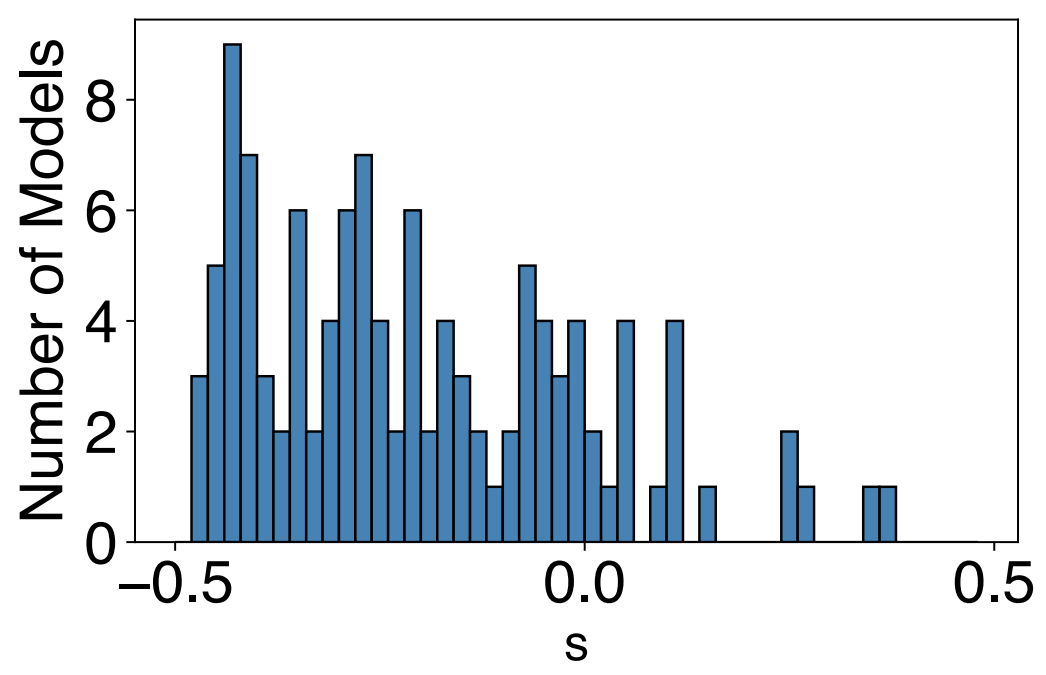

Figure S4: Separation measure, $\mathbf{s}$, of $114 \mathrm{MD}$ simulations, at temperatures above where structure formation occurs. $\mathbf{s}$ is defined as the number of like-contacts minus the number of non-like-contacts divided by the total number of contacts. A value of $\mathbf{s}=-1$ signifies only pure contacts (pure oligomers), and $\mathbf{s}=1$ signifies only non-like-contacts (racemic oligomers).

1.6 Charge-like Interactions: Our model treats molecular interactions very schematically and includes only attractive interactions of different strength. To understand the effect of explicit repulsive interactions, which occur for instance between functional groups of the same charge, we have performed a limited number of simulations of a model with such 'charge-like' interactions. In this model, molecules are imagined to contain two positively charged functional groups, two negatively charged groups, and one charge-neutral group, as illustrated in in Figure S5b. The functional form of these pair interactions is based on our purely attractive model described in the methods section of the main manuscript. For pairs of beads that are attractive (i.e., functional groups that are oppositely charged) we use $\epsilon_{\text {att }}=5 \epsilon$. For interactions between functional groups of like charge, we use $\epsilon_{\text {att }}=-5 \epsilon$; for interactions with charge-neutral beads we use $\epsilon_{\text {att }}=0$. As shown in Figure S5a, both like-charge and neutral interactions are purely repulsive, but likecharge interactions have a longer range, resulting in an energy penalty for nearest-neighbor contacts. Crystallization simulations were initiated from dispersed configurations of 5184 molecules at a density of 0.01 molecules $/ \sigma^{2}$ and were performed in the NVT ensemble at different manually adjusted temperatures using Langevin dynamics. Simulations were visually inspected for crystallization behavior. Out of all possible combinations of molecular shape and interactions, we simulated the crystallization of 76 randomly chosen models. Out of these, 5 models clearly formed enantiopure polymorphs and 4 models clearly formed racemic polymorphs. Furthermore, 7 models showed clear enantiopure tendencies, if not well-developed polymorphs, and 3 models formed racemic aggregates with semi-crystalline character. Examples of racemic and enantiopure polymorphs are given in Figure S6. The overall crystallization propensity of these models, as well 
as the fraction of enantiopure and racemic crystals, is therefore comparable to the purely attractive model analyzed throughout the main manuscript. It is therefore reasonable to assume that conclusions drawn in the main manuscript apply also to this, perhaps more realistic molecular model. However, a detailed analysis of the thermodynamic landscape of polymorphs for these "charged" models using POLYNUM is unfortunately complicated. Nearest-neighbor contacts between repulsive functional groups are largely avoided in the crystal structures of these models. As a result, polymorphs tend to have noticeably smaller packing fractions, as illustrated in Fig. S7a. The numerical effort of enumerating molecular packings increases dramatically with the amount of empty space in the lattice. In addition, several polymorphs violate the hexagonal lattice packing assumed by POLYNUM. An example of such an "off-lattice" polymorph is shown in Fig. S7b. A thermodynamic analysis of polymorphs is therefore beyond the scope of the current work.

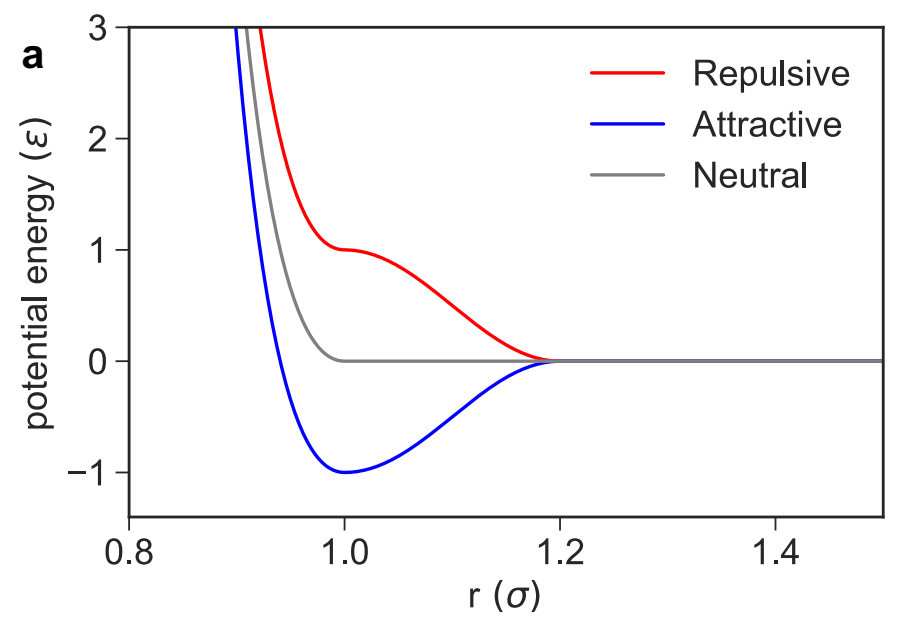

b
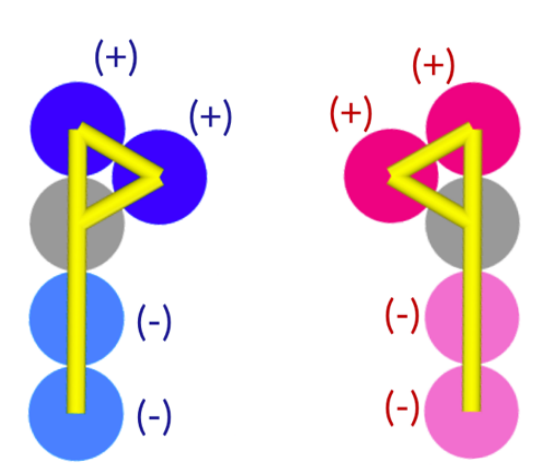

Figure S5: Charge-like interactions; (a) Pair potential used for like-charge (red line), opposite-charge (blue line), and neutral (grey line) interactions. (b) Schematic diagram of enantiomers of shape s6, each containing two positively charged beads, two negatively charged beads, and one neutral bead.

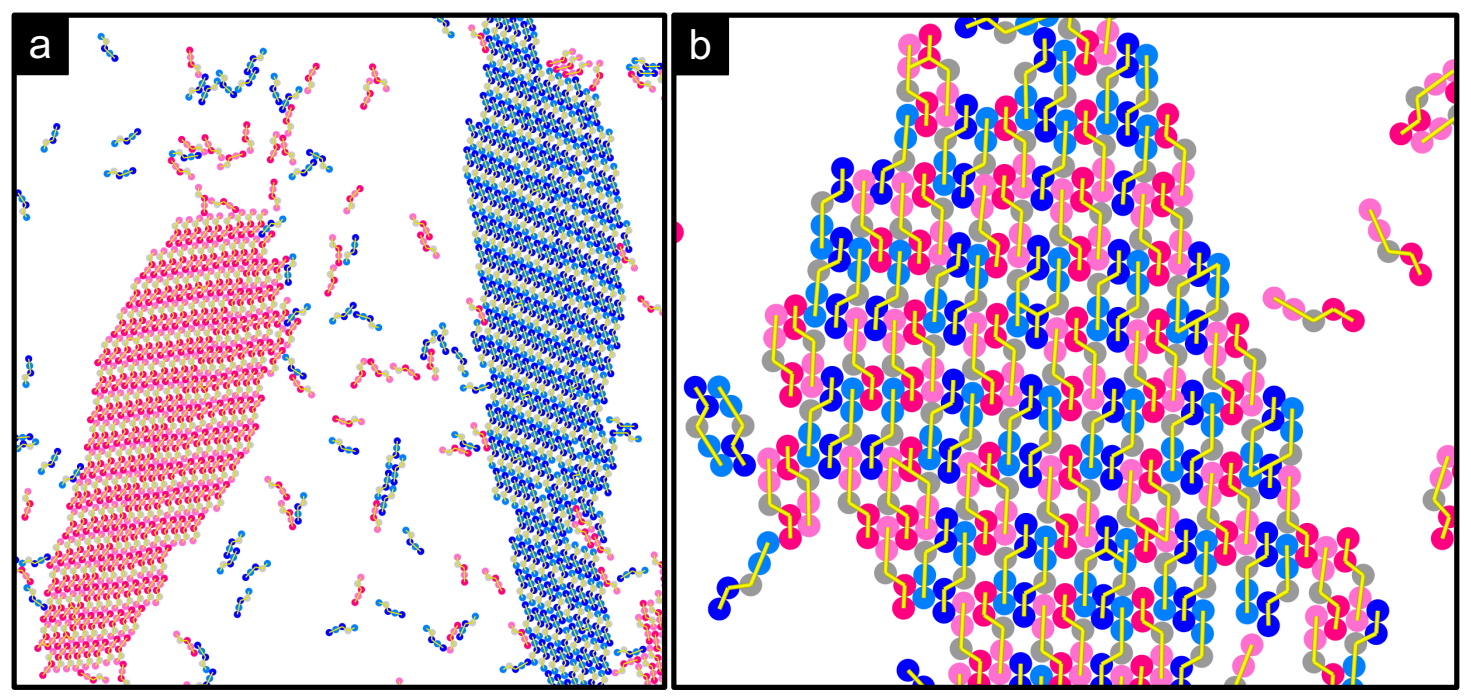

Figure S6: Examples of polymorphs obtained with models with charge-like interactions; (a) enantiopure, (b) racemic. 


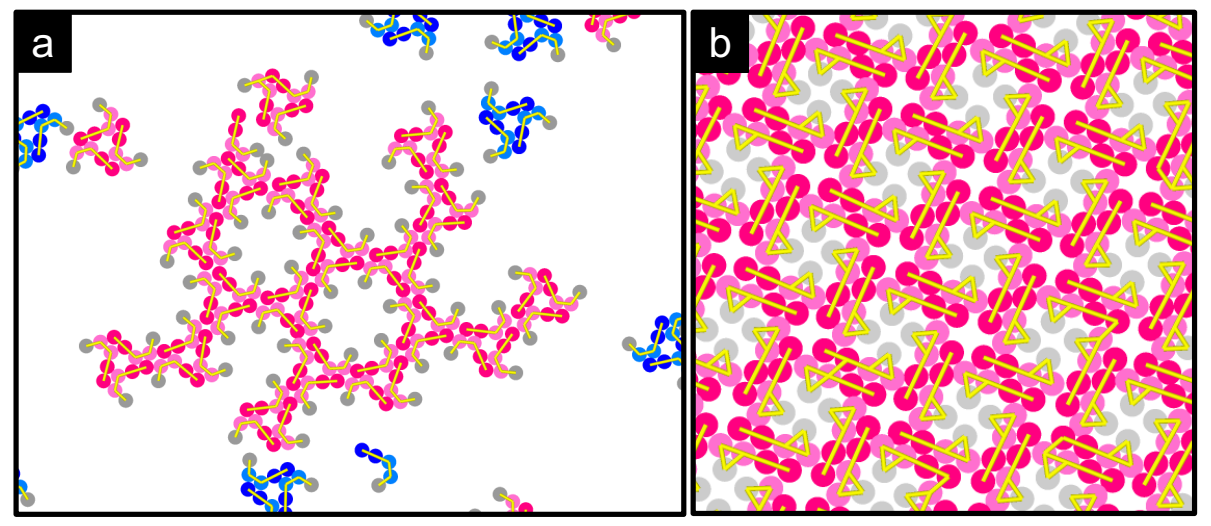

Figure S7: Characteristics of polymorphs obtained with "charged" model; (a) example of an 'open' polymorph, which cannot be readily obtained with POLYNUM; (b) example of a polymorph with positions of functional groups that do not coincide with the sites of a simple hexagonal lattice. 


\section{Part 2. POLYNUM}

2.1 Algorithm Overview: We have developed a crystal prediction algorithm that is capable of enumerating all the dynamically relevant crystal polymorphs of our model and their corresponding energies up to a unit cell size of 14 molecules and 4 holes. Our polymorph enumeration algorithm relies on a computer algorithm made popular by Donald Knuth, known as 'Algorithm X'. ${ }^{1}$ Algorithm $\mathrm{X}$ is a depth-first backtracking algorithm used for solving exact cover problems, such as sudoku puzzles and tiling problems. The implementation of this algorithm is called 'Dancing Links' and is described in the same paper. ${ }^{1}$

The general idea behind using 'Dancing Links' as a crystal prediction algorithm is to treat a unit cell as a period box that is then 'tiled' with a given chiral shape and its enantiomer, thus producing all possible polymorphs compatible with that unit cell-see Figure S8. By repeating this procedure for all unique unit cells, all possible polymorphs can in principle be enumerated. Of course, in practice the algorithm can only be carried out up to a maximum unit cell size, at which point the number of solutions becomes too large and the algorithm intractable.

A Java implementation of the 'Dancing Links' algorithm can be found at the following website: https://github.com/benfowler/dancing-links

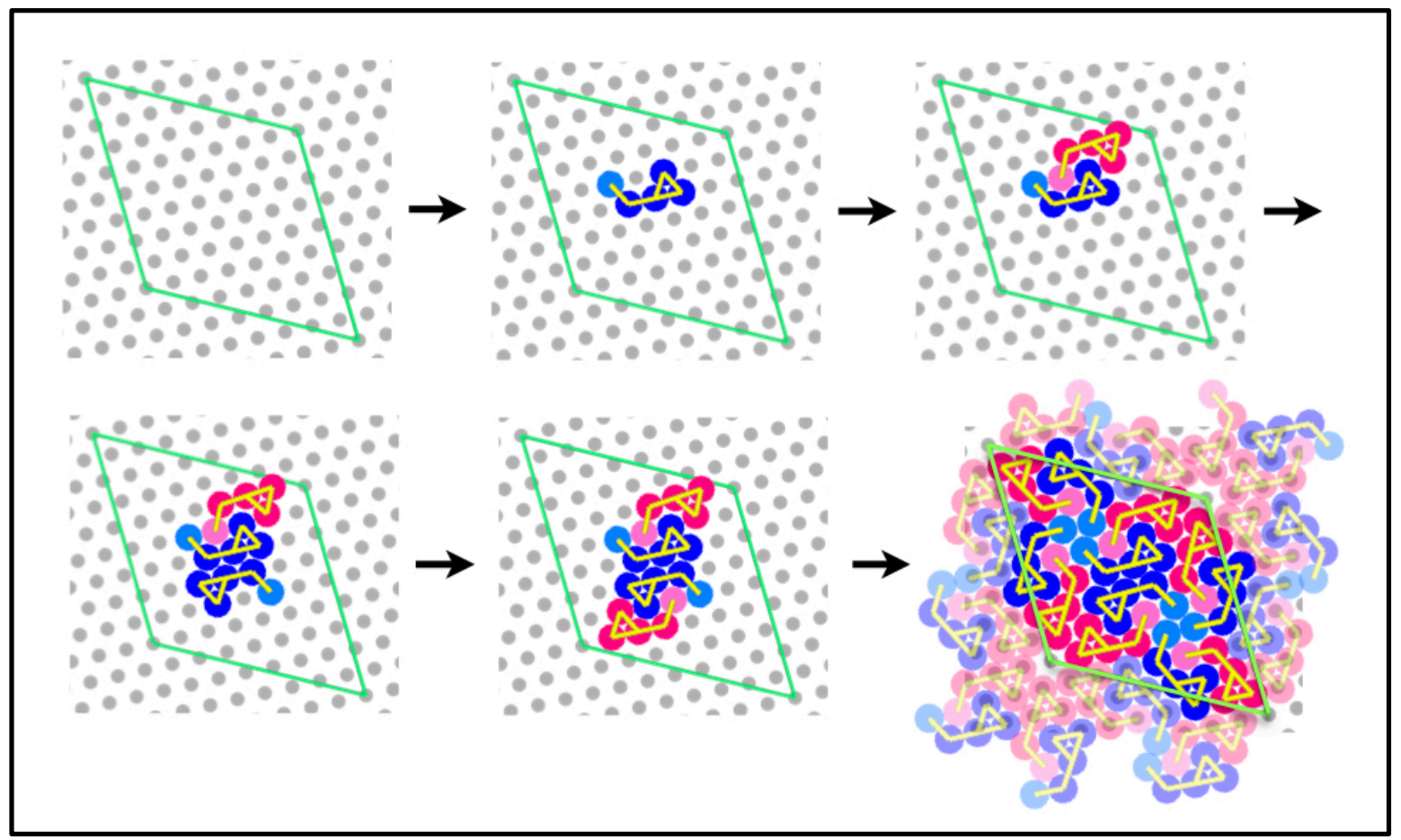

Figure S8: Schematic representation of how a unit cell is tiled by the 'Dancing Links' algorithm to produce a polymorph. Molecules with transparency are periodic images of molecules within the box.

The strength of this algorithm is also its primary computational limitation-since it enumerates all possible solutions for a given problem, the execution time increases exponentially with the size of the unit cell and the number of shapes. As a result, the algorithm quickly becomes intractable 
beyond a particular unit cell size. Fortunately, for the two-dimensional systems considered here, this size limit is sufficiently large to include the vast majority of relevant polymorphs. Since most of the crystals we find in MD simulations have unit cells containing 6 or fewer molecules, 'Dancing Links' is ideal for our purposes. A description of the algorithmic steps involved in POLYNUM is given next.

2.2 Unit Cell Determination: Unit cells were generated by enumerating all possible parallelograms whose corners terminate on the lattice sites of a hexagonal lattice and contain at least 5 (corresponding to a single molecules) and at most 75 lattice sites (15 molecules). Unit cells with any interior angle of less than $\pi / 3$ radians were removed, as we find that all of these unit cells are equivalent to other less skewed unit cells within the set-see Figure S9 for an example. Unit cells that are chiral enantiomers of other unit cells within the set were removed. Unit cells which are more skewed representations of other unit cells within the set were likewise removed, as were unit cells with aspect ratio larger than 2.75 . The final number of unit cells considered was 554.

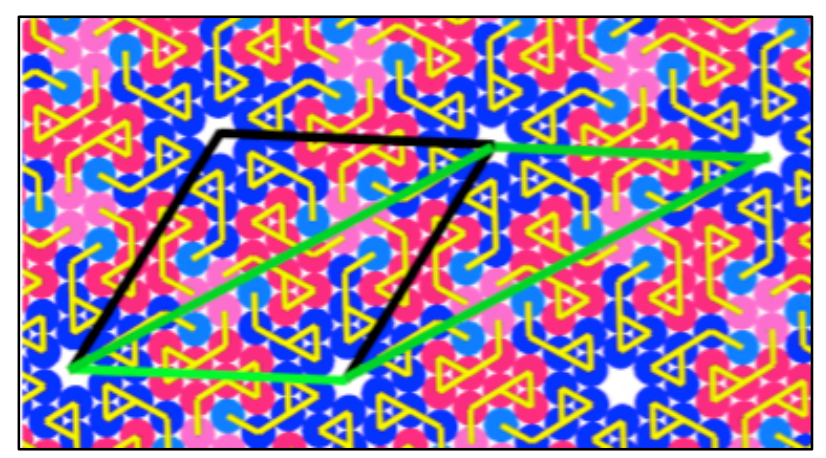

Figure S9: Example of a unit cell with an interior angle of less than $\pi / 3$ radians (green). The cell is equivalent to a the unit cell shown in black color, which has a larger interior angle.

2.3 Constructing an Incidence Matrix: The 'Dancing Links' algorithm operates on a so-called 'incidence matrix', which is comprised entirely of 0 s and $1 \mathrm{~s}$, representing all possible placements of molecules and holes in the unit cell. A complete description of how the 'Dancing Links' algorithm operates on the incidence matrix to produce tiling solutions is given in the paper by Knuth. ${ }^{1} \mathrm{~A}$ graphical description on how an incidence matrix is generated for POLYNUM is given in the accompanying file: DancingLinksForPolymorphEnumeration.pdf.

2.4 Removing Equivalent Solutions: The procedure described above produces many translationally and rotationally equivalent solutions in the same unit cell. In addition, solutions for different unit cells might also be equivalent. For example, a unit cell of 12 molecules will contain many solutions that can be represented by smaller unit cells, (i.e., containing 1, 2, 3, 4, and 6 molecules). We developed an efficient way of eliminating equivalent solutions within a unit cell and across different unit cells. For each solution (polymorph) found by POLYNUM, we calculate two objects: the contact vector and a unique tag.

2.4.1 Contact vector: As described in the main manuscript, the contact vector $\vec{c}_{p}$ contains the number of contacts made between different functional groups in a unit cell divided by 
the number of molecules in the unit cell. This contact vector is used in our first check to determine if two polymorphs are equivalent. If two polymorphs have different contact vectors, they must be different. But because two different polymorphs can have the same contact vector, an additional criterion is required to identify duplicate polymorphs.

2.4.2 Unique Tags: Unique tags contain information about the spatial patterns formed by different types of functional groups contained in a polymorph. These tags are constructed as follows.

1. First, the functional groups of different enantiomers in a polymorph are given IDs according to the following convention: for a left-handed molecule we use bead IDs 1-5; IDs 6-10 are used for right-handed molecules. The sequence of IDs encountered by traversing the unit cell in one of three unique directions of the hexagonal lattice is stored. Only the shortest repeating sequence is considered. Repeat for the remaining 2 directions.

Example: $[2,3,4,5,1,2,3,4,5,1,2,3,4,5,1]$ is truncated to $[2,3,4,5,1]$

2. A lexicographically minimal string rotation operation ${ }^{2}$ is performed on the sequences of bead numbers.

Example: $[2,3,4,5,1]$ is converted to $[1,2,3,4,5]$.

3. The sequences generated along each of the 3 unique directions are combined according to their lexicographical order.

$$
\begin{aligned}
& \text { Example: } \quad[1,3,2,4,5], \\
& [1,2,3,4,5,1,3,2,4,5,6,7,8,9,10]) .
\end{aligned} \quad[1,2,3,4,5], \quad[6,7,8,9,10] \quad \rightarrow
$$

4. To account for the chiral enantiomer polymorph of each polymorph, the sequence of numbers is doubled, by combining, according to lexicographical order, the previously found sequence of numbers with a transposed set of numbers. (Numbers 1-5 are replaced with 6-10 and numbers 6-10 are replaced with 1-5)

$$
\begin{gathered}
\text { Example: } \begin{array}{c}
{[1,2,3,4,5,1,3,2,4,5,6,7,8,9,10] \rightarrow} \\
{[1,2,3,4,5,1,3,2,4,5,6,7,8,9,10,}
\end{array} \\
6,7,8,9,10,6,8,7,9,10,1,2,3,4,5]) .
\end{gathered}
$$

5. The sequence of numbers is converted into a string, i.e., integers 1-10 are converted to letters $a-j$

Example: $[1,2,3,4,5,1,3,2,4,5,6,7,8,9,10,6,7,8,9,10,6,8,7,9,10,1,2,3,4,5] \rightarrow$ 'abcdeacbdefghijfghijfhgijabcde'

The so-constructed unique identifying strings can be compared with fast algorithms, allowing each new generated polymorph to be compared against a set of millions of polymorphs with relatively small computational cost. This procedure is considerably more efficient than bond order diagrams ${ }^{3}$ or other similar measures. Also, 'tags' are invariant to the size of the unit cell (i.e., a polymorph unit cell which can be 
subdivided into smaller unit cells will receive the same identifying tag as one of the smaller subunits) as well as chiral enantiomer polymorphs (polymorphs which are chiral enantiomers of one another will receive the same tag). Although we cannot strictly show that identifying tags are unique, we have not found any examples of two different polymorphs with the same tag.

\subsection{Examples of Polymorph Solutions:}

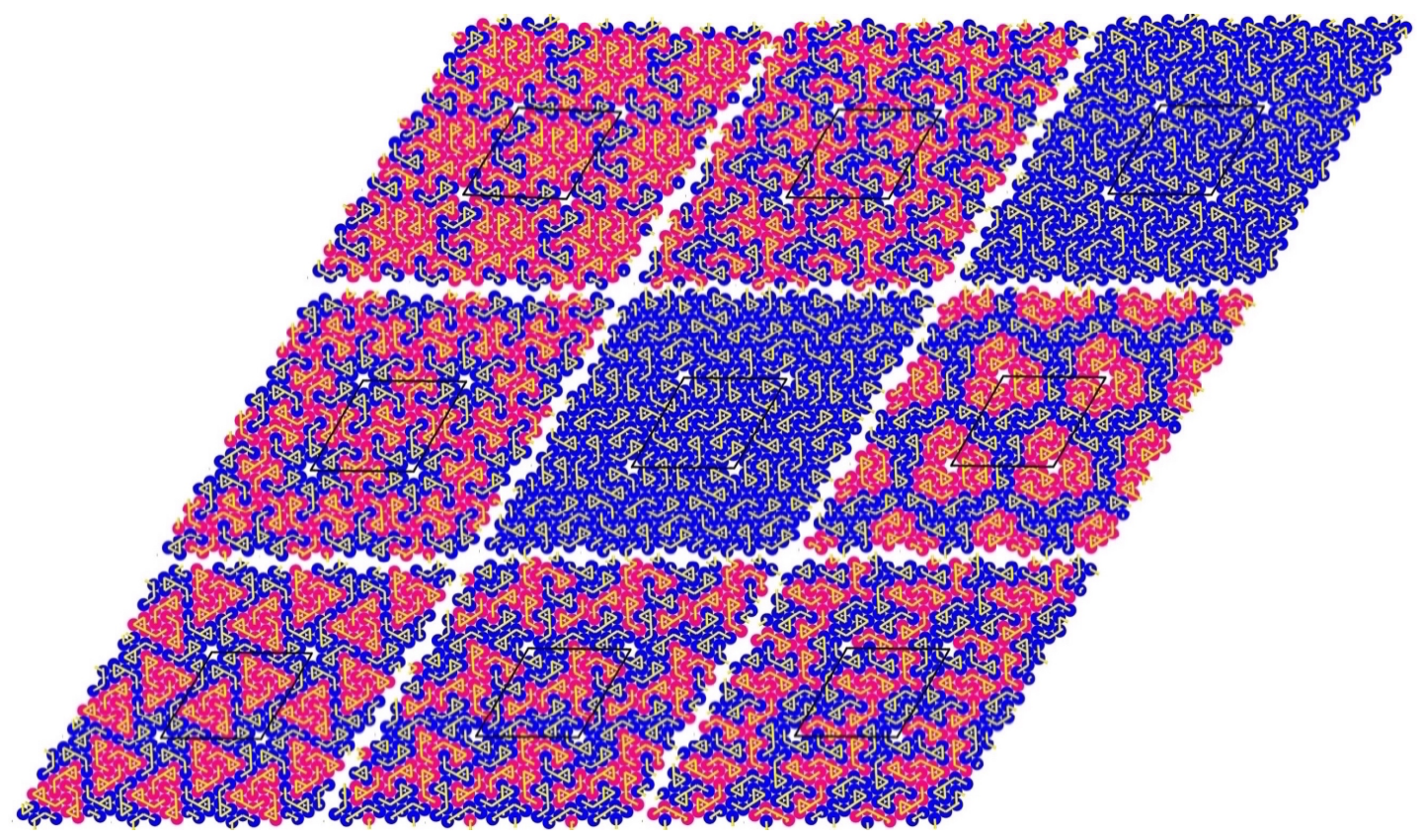

Figure S10: Examples of different polymorphs of shape s2, for a unit cell containing 12 molecules and one hole.

\subsection{Exponential Increase in Polymorph Solutions with Added Holes:}

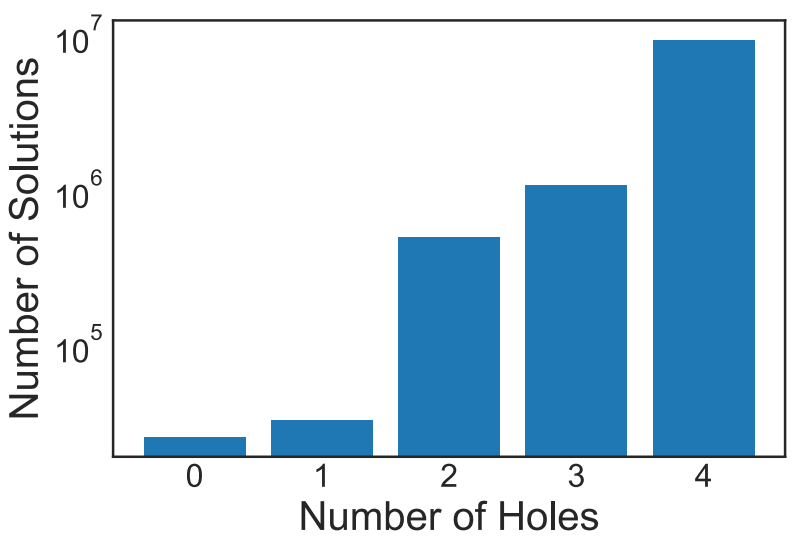

Figure S11: Number of unique polymorphs found for all shapes in unit cells containing 8 molecules, as a function of the number of holes (i.e., unoccupied lattice sites) in the unit cell. 
2.7 Verification of Crystallinity Algorithm and Polymorph Enumeration Procedure: In order to determine if our POLYNUM calculations are able to capture all dynamically relevant polymorphs, all MD simulations of racemic solutions using an interaction strength of $\epsilon_{s}=5 \epsilon$ were manually inspected for crystalline clusters. We did not find any large additional crystalline clusters that were not already identified by our crystallinity determination algorithm (described below). Each crystalline cluster shown in Figure 2 of the main text was compared to the set of low energy polymorphs determined by POLYNUM. Figure S12 shows the polymorphs that correspond to the crystalline clusters found in each MD simulation.

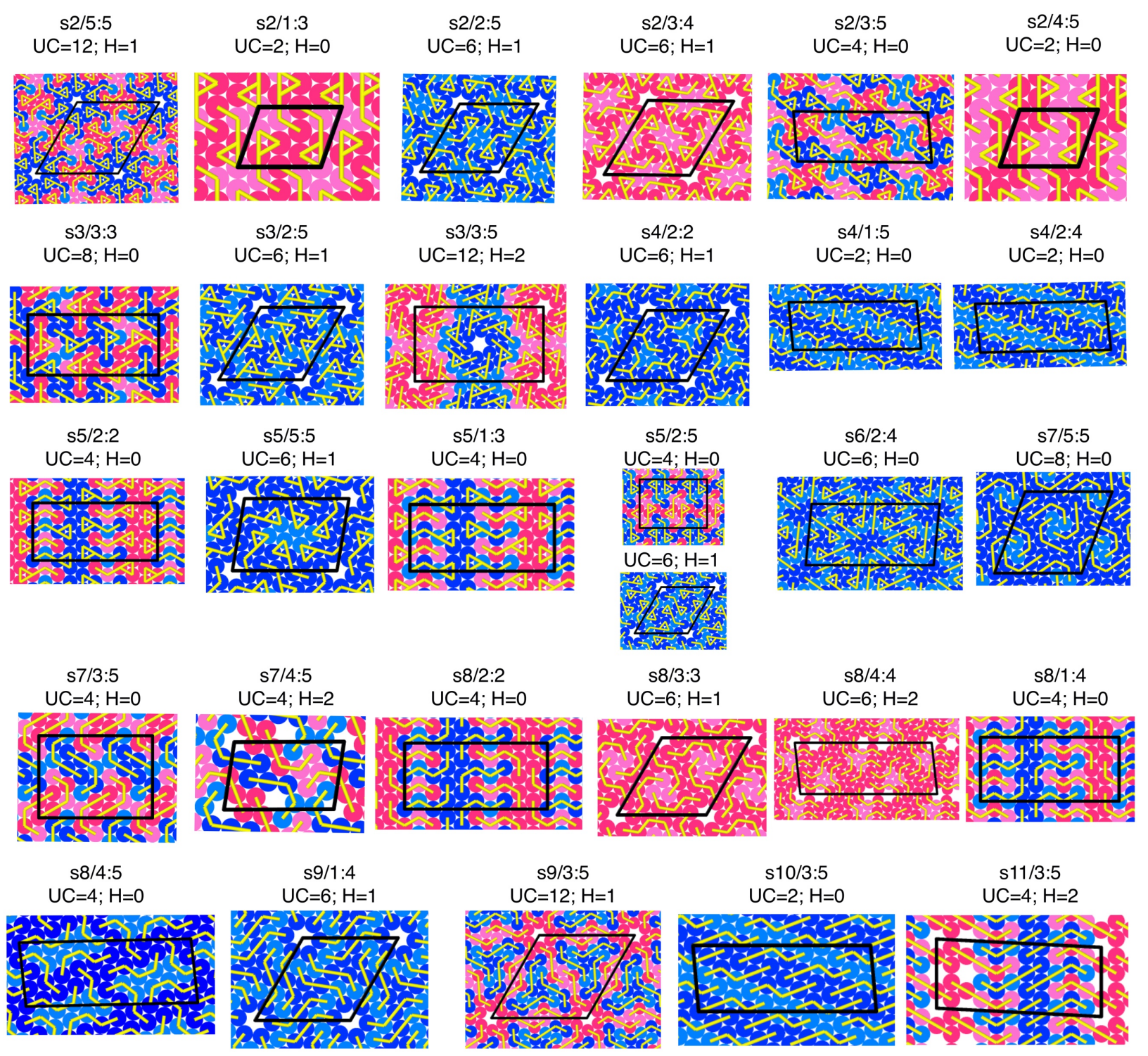

Figure S12: Pictures of polymorph unit cells, identified by POLYNUM, that match the crystalline clusters found in MD simulations of racemic solutions with $\epsilon_{s}=5 \epsilon$. Note that the black outline does not necessarily represent the smallest possible unit cell for each polymorph. In all cases we specify the unit cell size (UC) and number of holes $(H)$. 
In all cases but one, the set of polymorphs enumerated by POLYNUM clearly contained the crystal polymorph observed in the simulation. In one case (s7/5:5), the assignment of a unit cell to the cluster in the MD simulation was ambiguous because of the partial disorder present in the cluster. However, POLYNUM found many polymorphs that contain very similar motifs (namely, arrangements of micellular trimers and intermittent holes)-see Figure S13. This leads us to conclude that even if dynamically relevant polymorphs with very large unit cells are missing from our POLYNUM analysis, a similar but more modest-sized polymorph will likely be found. Furthermore, as can be seen from Figure S12, the majority of polymorphs that form in simulations have unit cells containing 6 molecules or fewer with 0 or 1 hole. We find the largest ratio of holes to molecules for $s 7 / 4: 5$, with 2 holes for a unit cell size of 4 molecules.

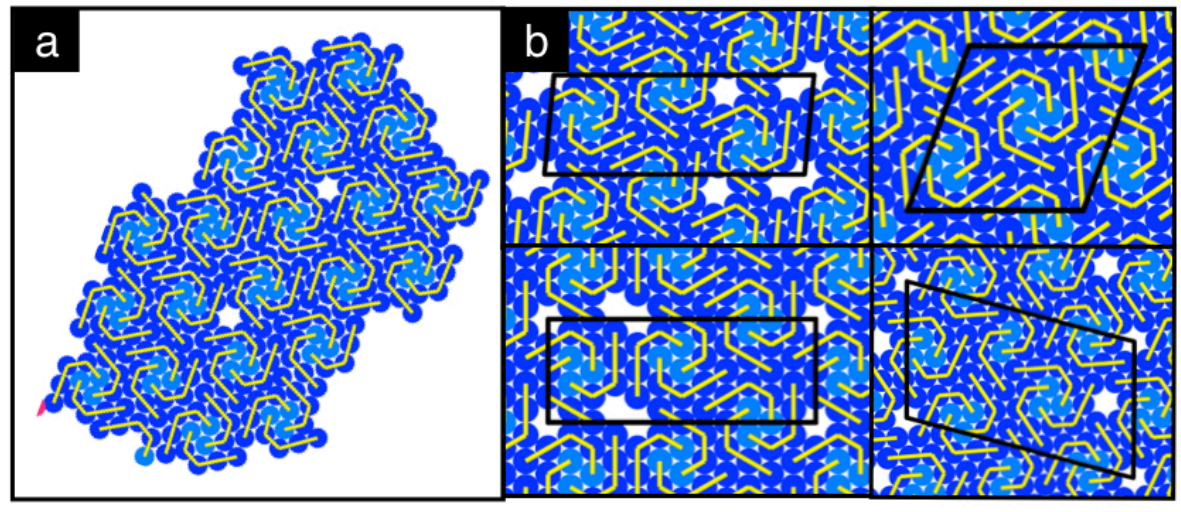

Figure S13: (a) the crystalline cluster observed in the MD simulation with parameters $s 7 / 5: 5 / 5$. (b) Calculated polymorphs containing very similar motifs to the cluster found in (a).

\subsection{Increase in Energy of Polymorphs as a Function of Packing Fraction:}

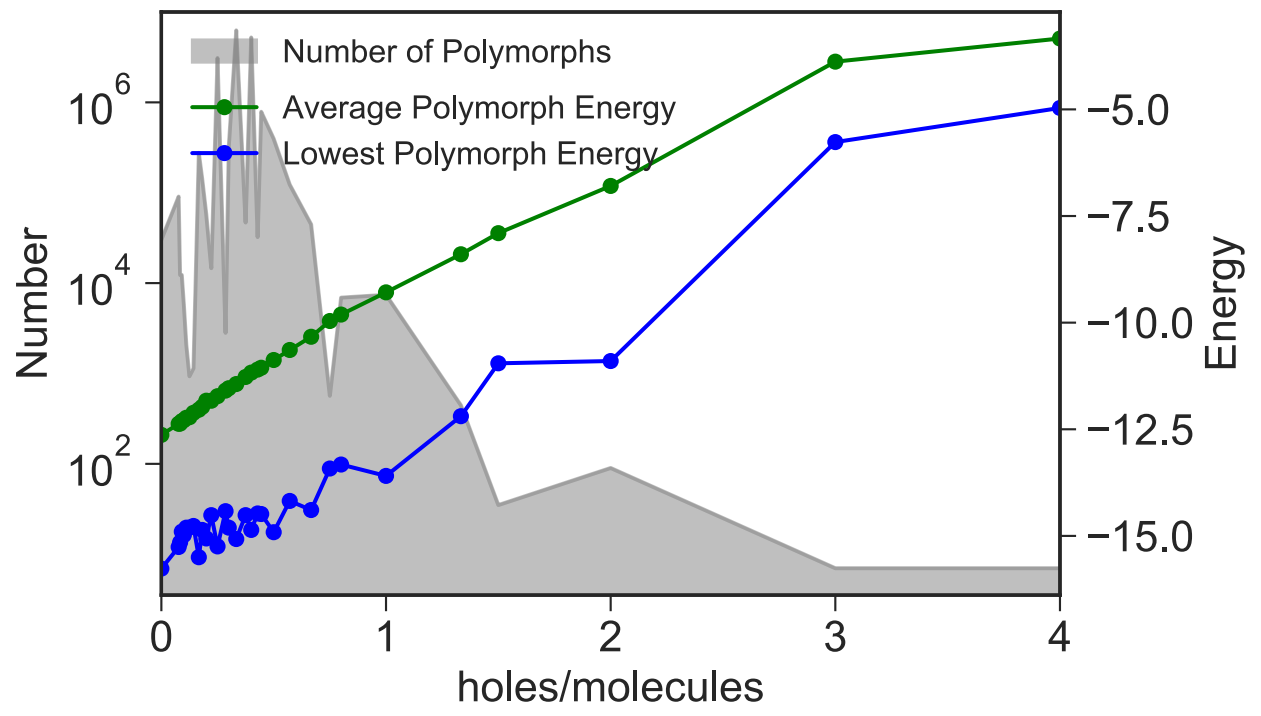

Figure S14: (left axis) The number of polymorphs computed for shape s2 as a function of packing fraction (i.e. number of holes divided by the number of molecules in the unit cell). (right axis) The average and lowest energy polymorphs found at a given packing fraction for molecule s2, averaged across all interaction types where $\epsilon_{s}=5 \epsilon$. 


\section{Part 3. Determining Crystalline Quality (CQ)}

3.1 Validation: In order to verify that our crystallinity determination algorithm was sufficiently accurate in identifying polymorphs in simulations the following procedure was performed: A set of 160 randomly chosen polymorphs were simulated in two ways: as periodic bulk crystals and as large (but finite) clusters in a simulation box. Each simulation contained roughly 1000 molecules and was performed at a temperature of $T=1.0 \frac{\epsilon}{k_{B}}$. Results were then analyzed using our crystallinity determination algorithm to (1) identify the polymorph, and (2) determine a CQ score. Our algorithm identified the simulated polymorph with $98.7 \%$ and $97.5 \%$ accuracy for the periodic crystals and large clusters, respectively. Average CQ scores were 0.985 and 0.835 in the periodic crystals and large clusters, respectively.

\subsection{Examples from MD Simulations:}

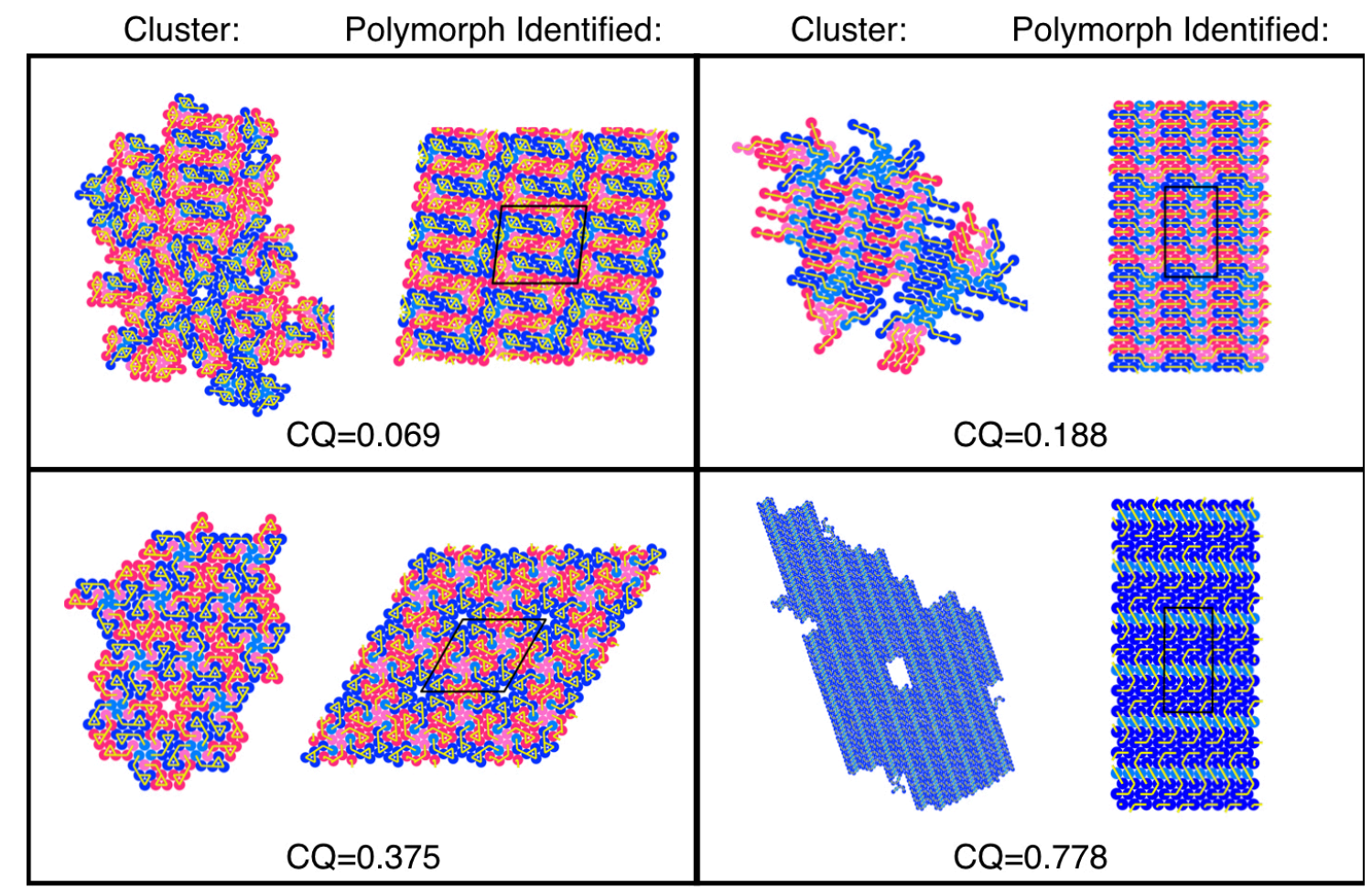

Figure S15: Examples of clusters drawn from MD simulations along with identified polymorphs and $C Q$ scores. 


\section{Part 4. Free Energy Calculations}

4.1 Procedure: In order to verify that zero-temperature energy differences between polymorphs are good estimates for free energy differences at the temperatures of crystal formation, we performed free energy calculations on three sets of polymorphs of different molecules. The lattice coupling/expansion method ${ }^{4}$ was used to calculate free energies, using the following parameters:

- Temperatures were set to the same values at which crystal growth was observed in MD simulations of solutions of monomers.

- Equilibrium volumes were determined by running NPT simulation at 0 pressure for 200,000 time steps.

- 100 separate simulations were performed at different harmonic potential scaling factors ( $\lambda$ ), from $\lambda=0.001$ to $\lambda=1.0$, in the NVT ensemble, for 200,000 time steps each.

- 200 separate simulations were performed at different volume scaling factors $(\gamma)$, from $\gamma=1$ to $\gamma=2$ (at which point molecules were non-interacting), in the NVT ensemble, for 200,000 time steps each.

- Three independent free energy determinations were performed for each polymorph studied.

4.2 Results: The results of the free energy calculations are show in Table S3. The absolute differences between $0 \mathrm{~K}$ energy differences and free energy differences at crystallization temperatures range from $0.03 \varepsilon$ to $0.19 \varepsilon$ per molecule. Note that the polymorph that forms in MD simulations has larger free energy than the lowest energy polymorph in all cases studied. This leads us to the conclusion that entropic corrections to OK energies can be neglected on our models.

\begin{tabular}{|c|c|c|c|c|c|c|c|}
\hline Shape & Interaction & FE (Temp) & Polymorph & $\overline{F E}$ & $+/-$ & OK Energy & $\overline{O K}-\mathrm{FE}$ \\
\hline \multirow{3}{*}{ s9 } & \multirow{3}{*}{$1: 4$} & \multirow{3}{*}{$1.15 \mathrm{k}_{\mathrm{B}} / \varepsilon$} & $\bar{A}$ & 0.00 & 0.006 & 0.00 & - \\
\hline & & & B & -0.53 & 0.008 & -0.50 & 0.03 \\
\hline & & & C & -0.55 & 0.003 & -0.50 & 0.05 \\
\hline \multirow{2}{*}{ s6 } & \multirow{2}{*}{$2: 4$} & \multirow{2}{*}{$1.05 \mathrm{k}_{\mathrm{B}} / \varepsilon$} & D & 0.00 & 0.011 & 0.00 & - \\
\hline & & & $E$ & -0.12 & 0.009 & -0.17 & -0.05 \\
\hline \multirow{5}{*}{ s4 } & \multirow{5}{*}{$1: 5$} & \multirow{5}{*}{$1.10 \mathrm{k}_{\mathrm{B}} / \varepsilon$} & $F$ & -0.17 & 0.007 & 0.00 & 0.17 \\
\hline & & & G & -0.06 & 0.006 & 0.00 & 0.06 \\
\hline & & & $\mathrm{H}$ & 0.00 & 0.006 & 0.00 & - \\
\hline & & & I & -0.19 & 0.008 & 0.00 & 0.19 \\
\hline & & & $J$ & -0.15 & 0.006 & 0.00 & 0.15 \\
\hline
\end{tabular}

Table S3: Results of free energy calculations for 3 sets of polymorphs, given an interaction strength of $\epsilon_{S}=5 \epsilon$. For each set, the polymorph which forms in MD simulations is highlighted in red. All free energies and OK energies are reported relative to the highest energy polymorph in each set. 


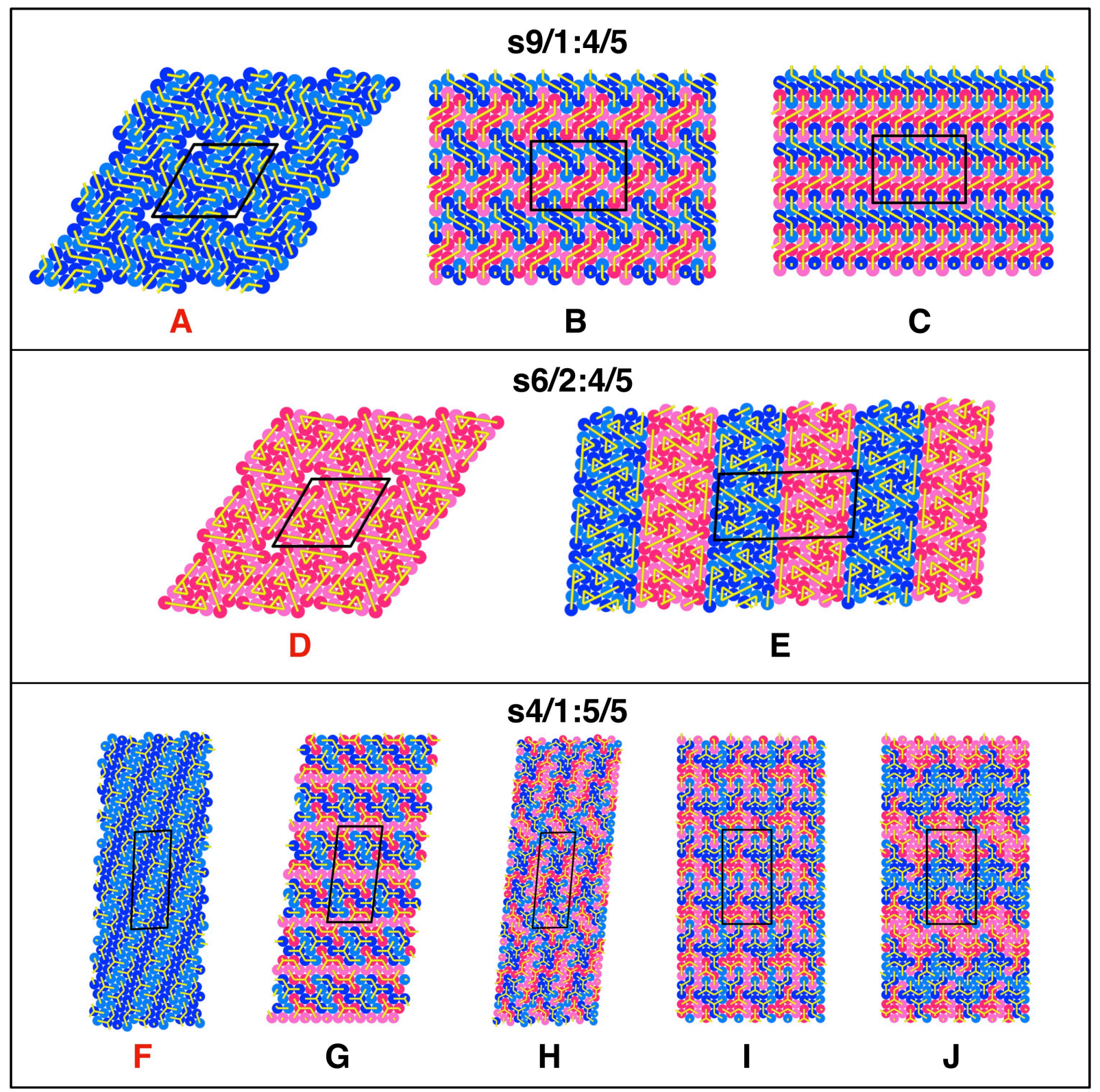

Figure S16: Images of polymorphs for which free energy calculations were performed, A$\mathrm{J}$, as are described in Table S3. The polymorph which forms in MD simulations is highlighted in red. 


\section{Part 5. Competing Polymorphs}

5.1. Effects of Orientational Constraints on Crystallization and Separation Propensity: In a recent paper by Woszczyk and coworkers, the authors showed that constraining the orientations of $2 \mathrm{D}$ chiral molecules results in an increased propensity for crystallization and chiral separation. In that study, MC simulations were performed on the same set of molecular shapes used in this work but with completely uniform interactions $\left(\epsilon_{s}=\epsilon\right)$. As an example, we consider the crystallization behavior of shape 8 in enantiopure and racemic solutions, both with and without orientational constraints. Without orientational constraints and in an enantiopure solution, s8 forms a semi-crystalline arrangement. With orientational constraints, the same solution forms large crystalline domains. In a racemic solution without orientational constraints, a completely disordered mixture is observed. With orientational constraints, the solution produces an ordered crystal. Using the ideas developed in the main text of this paper, we argue that the observed behavior is cause by dramatic changes of the number of competing polymorphs upon introduction of orientational constraints. Applying an orientational constraint effectively removes large numbers of polymorphs, strongly reducing the number of competing polymorphs. Using the set of polymorphs enumerated for s8 with POLYNUM, we are able to compute the number of dynamically relevant polymorphs with and without orientational constraints. Table S4 shows the result of this calculation.

\begin{tabular}{|l|c|c|}
\hline & \multicolumn{2}{|c|}{ Solution Type } \\
\hline $\mathbf{N}_{0.95}$ & Pure & Mixed \\
\hline No Confinement & 136 & 6644 \\
\hline w/ Confinement & 1 & 4 \\
\hline All Polymorphs & & \\
\hline No Confinement & 2866 & 178187 \\
\hline w/ Confinement & 20 & 35 \\
\hline
\end{tabular}

Table S4: Number of competing polymorphs of s8, with and without orientational constraints.

As evident from the table, the total number of polymorphs found for $\mathrm{s} 8$ in racemic solutions was 178,187 . Requiring that molecules be confined to one particular orientation reduces this number to only 35. The number of dynamically competing polymorphs in the racemic solution without orientational constraints is 6,644 . With orientation constraints, this number is reduced to 4 . Figure S17 shows two competing polymorphs at the same energy, which are manifest in the MC simulations by Woszczyk and coworkers. 


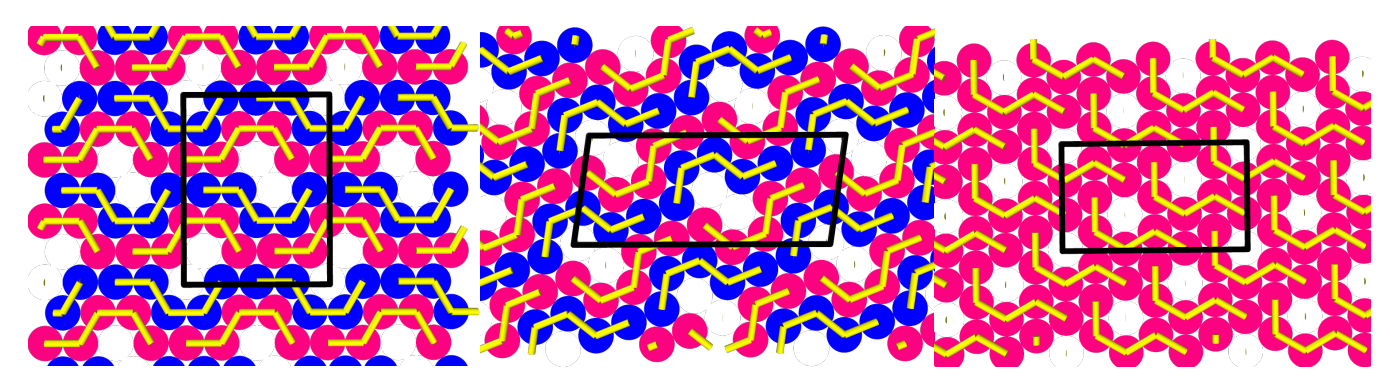

Figure S17: Left \& Middle: Two competing racemic polymorphs at the same energy. Right: The lowest energy pure polymorph has no competitors.

\subsection{Enantiomer Ratios vs Binomial Statistics:}

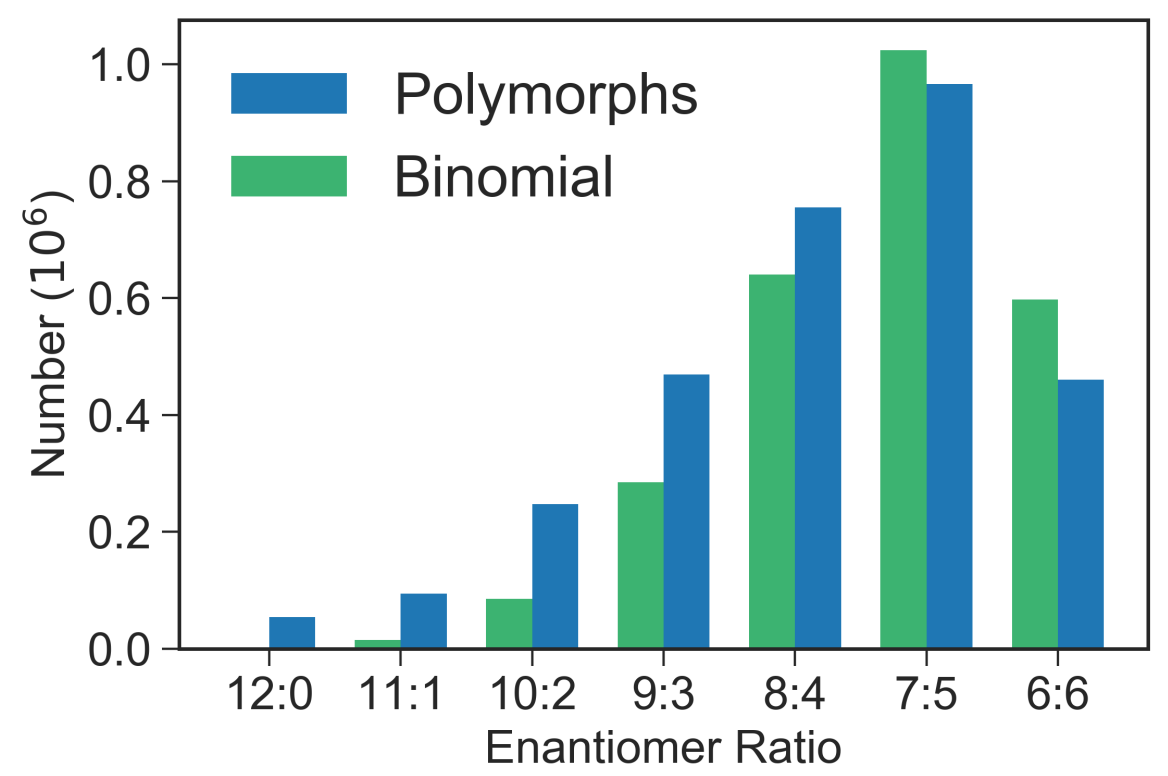

Figure S18: Comparison of a best fit binomial distribution (green) and the numbers of polymorphs (blue) with different number ratios of left- and right-handed molecules in the unit cell, found for shape $s 9$ and all unit cells containing 12 molecules and no holes. Chiral enantiomers of unit cells are included in each group, i.e., the group with enantiomer ratio of 12:0 (enantiopure) contains polymorphs exclusively composed of left- and right-handed molecules, respectively. In that sense, the group labeled "11:1" also contains all polymorphs of composition "1:11", and so on. "6:6" represents all racemic polymorphs. All other ratios represent polymorphs with fractional composition (PFCs). 


\section{Part 6. Predicting Crystallization Using Machine Learning Techniques}

Summary: Using machine learning techniques, we have performed additional data analysis and simulations to corroborate the relation between interaction strength and polymorph distributions described in the main paper. Our main results include:

1. For a given molecular shape, the map between interaction vector $\vec{\epsilon}$ and energy difference $\Delta E$ between lowest-energy pure and racemic polymorphs can be successfully learned by a neural network (NN) to high accuracy. This result suggests that the underlying polymorph space that determines $\Delta E$ has a relatively simple structure. Further analysis showed that the number of enantiopure and racemic polymorphs that contribute to $\Delta E$ is surprisingly small.

2. We used the $\mathrm{NN}$ described above to select $\vec{\epsilon}$ that result in a large thermodynamic advantage of enantiopure crystals (i.e., $\Delta E / E_{0} \leq-0.1$ ). In nearly all cases, these models indeed spontaneously formed enantiopure crystals in molecular dynamics computer simulations, confirming that a strong thermodynamic bias is an excellent predictor of crystallization behavior.

3. Interaction vectors $\vec{\epsilon}$ that produced large negative or large positive $\Delta E$, as described above, could generally be grouped into a few distinct clusters using K-means ${ }^{5}$ or similar high-dimensional clustering algorithms. These interaction vectors were largely heterogeneous (i.e., large $\varphi_{\vec{c}_{p}}$ ) and clusters of interaction vectors were characterized by one or two strong interactions each.

4. We unsuccessfully attempted to learn $\Delta E$ across the set of 11 molecular shapes, using interaction vectors $\vec{\epsilon}$ and a set of numbers characterizing molecular shape as input. We trained several such NN, using data for 10 of the 11 molecular shapes as our training set, and testing the quality of the fit on the remaining shape. All such attempts resulted in large test set errors, indicating that the dependence of $\Delta E$ on molecular shape is likely complex and a much larger set of shapes would be needed to enable a good fit.

6.1. Training a $\mathrm{NN}$ to Learn $\Delta \mathrm{E}$ for Fixed Molecular Shape. We randomly generated a set of $10^{6}$ interaction vectors $\vec{\epsilon}$, whose 15 components $\epsilon_{i}$ are the strengths of interactions between all pairs of functional groups of a given molecule. Interaction vectors were generated under the constraint $\sum \epsilon_{i}=-19 \epsilon$, equivalent to interactions with $\epsilon_{S}=5 \epsilon$ discussed in the main manuscript, using the Bayesian Bootstrap method. ${ }^{6}$ For a given molecule, we calculated the energy gaps $\Delta E$ for all $10^{6}$ interaction vectors. The data was split into training and test sets of 800,000 and 200,000 interaction vectors, respectively. Neural networks ( 3 hidden layers, 128 nodes per layer) to predict $\Delta E$ were trained with the TensorFlow package using the components $\epsilon_{i}$ as input.

As demonstrated in Figure $\mathrm{S} 19 \mathrm{a}$, the $\mathrm{NN}$ predicting $\Delta \mathrm{E}$ with good accuracy. To investigate the complexity of the map between $\vec{\epsilon}$ and $\Delta \mathrm{E}$, we also performed a linear regression on the data. As shown in Table S5, the linear regression model is unable to accurately predict $\Delta \mathrm{E}$. However, our analysis suggests that many interaction vectors result in the same polymorphs at the lowest energies. In Figure S19b, we plot the fraction of randomly generated interaction vectors that produce the same lowest-energy polymorphs, for the 50 most important polymorphs. As evident from the figure, there is a particular enantiopure polymorph that has the lowest energy in $24 \%$ of all molecules with shape s8. Only 10 enantiopure and racemic polymorphs determine $\Delta \mathrm{E}$ for the 
majority of molecules. Note that these polymorphs span the "convex hull" of enantiopure and racemic polymorphs as introduced by Nicholls and coworkers. ${ }^{7}$
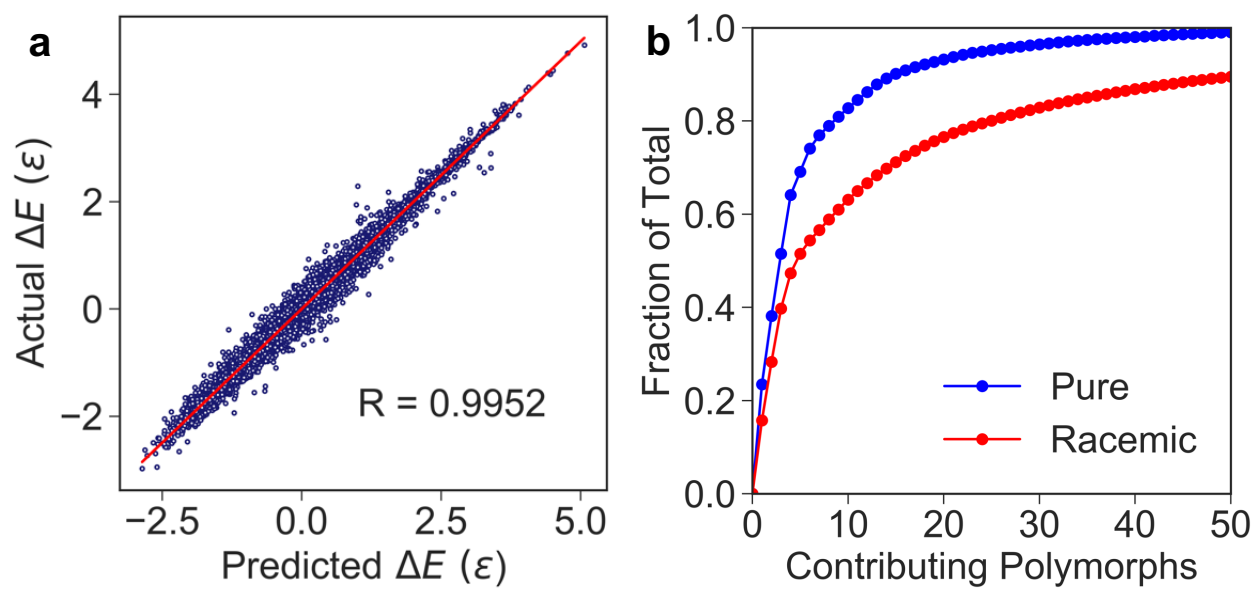

Figure S19. NN predictions of $\Delta E$ for molecular shape s8. (a) Predicted vs. actual $\Delta \mathrm{E}$ of 100,000 random interaction vectors using a NN. The red line is the best fit line to the data. (b) A plot of the fraction of $\Delta E$ vs. the number of enantiopure and racemic polymorphs whose energies determine $\Delta E$, across the same set of 100,000 molecules shown in (a).

\begin{tabular}{|c|c|c|c|c|c|c|c|c|c|c|c|c|c|c|c|}
\hline & $1: 1$ & $2: 2$ & $3: 3$ & $4: 4$ & $5: 5$ & $1: 2$ & $1: 3$ & $1: 4$ & $1: 5$ & $2: 3$ & $2: 4$ & $2: 5$ & $3: 4$ & $3: 5$ & $4: 5$ \\
\hline $\begin{array}{r}\text { Linear Model } \\
\Delta E(\epsilon)\end{array}$ & -0.29 & 0.22 & -0.09 & -0.50 & -0.08 & -0.19 & -0.16 & 1.12 & 0.50 & -0.38 & 0.90 & -0.24 & 0.08 & 1.09 & -0.61 \\
\hline $\begin{array}{r}\text { NN Model } \\
\Delta E(\epsilon) \\
\end{array}$ & -1.04 & 0.97 & 0.15 & -0.79 & 0.18 & 0.57 & -1.16 & 0.13 & -0.04 & 0.66 & 0.26 & -0.05 & 0.75 & 0.11 & $\mid-1.80$ \\
\hline $\begin{array}{l}\text { Actual } \\
\Delta E(\epsilon)\end{array}$ & -1.00 & 1.00 & 0.25 & -0.75 & 0.25 & 0.50 & -1.08 & 0.00 & 0.00 & 0.67 & 0.00 & 0.00 & 0.75 & 0.00 & $\mid-1.67$ \\
\hline $\begin{array}{r}\text { Linear Model } \\
\text { Error }(\epsilon) \\
\end{array}$ & 0.71 & -0.78 & -0.34 & 0.25 & -0.33 & -0.69 & 0.92 & 1.12 & 0.50 & -1.04 & 0.90 & -0.24 & -0.67 & 1.09 & 1.05 \\
\hline $\begin{array}{r}\text { NN Model } \\
\text { Error }(\epsilon) \\
\end{array}$ & -0.04 & -0.03 & -0.10 & -0.04 & -0.07 & 0.07 & -0.08 & 0.13 & -0.04 & -0.01 & 0.26 & -0.05 & 0.00 & 0.11 & -0.13 \\
\hline $\begin{array}{l}\text { Linear Model } \\
\text { Avg Error }(\epsilon)\end{array}$ & 0.71 & & & & & & & & & & & & & & \\
\hline $\begin{array}{r}\text { NN Model } \\
\text { Avg Error }(\epsilon)\end{array}$ & 0.08 & & & & & & & & & & & & & & \\
\hline
\end{tabular}

Table S5. Comparison of the quality of the fit obtained with a linear regression model and a NN for all simulated molecules of shape s8. Pairs of indices $(1: 1,2: 2$, etc.) indicate pairs of strongly interacting functional groups, as in the main manuscript. 
6.2. NN Predicts Molecules that Form Enantiopure Crystals in Simulations. To confirm that large thermodynamic preferences for enantiopure crystals are predictive of crystallization behavior, we randomly generated many interaction vectors and selected 20 models with $\Delta E / E_{0} \leq$ -0.1 . We were interested to see if interaction vectors predicted by the NN to have a large thermodynamic bias for enantiopure polymorphs would produce enantiopure crystallization under the same crystallization protocol as described in the text. As expected, nearly all of these models readily crystallized into enantiopure crystals of high quality in molecular dynamics computer simulations. Four examples of these crystals are shown in Figure S20.

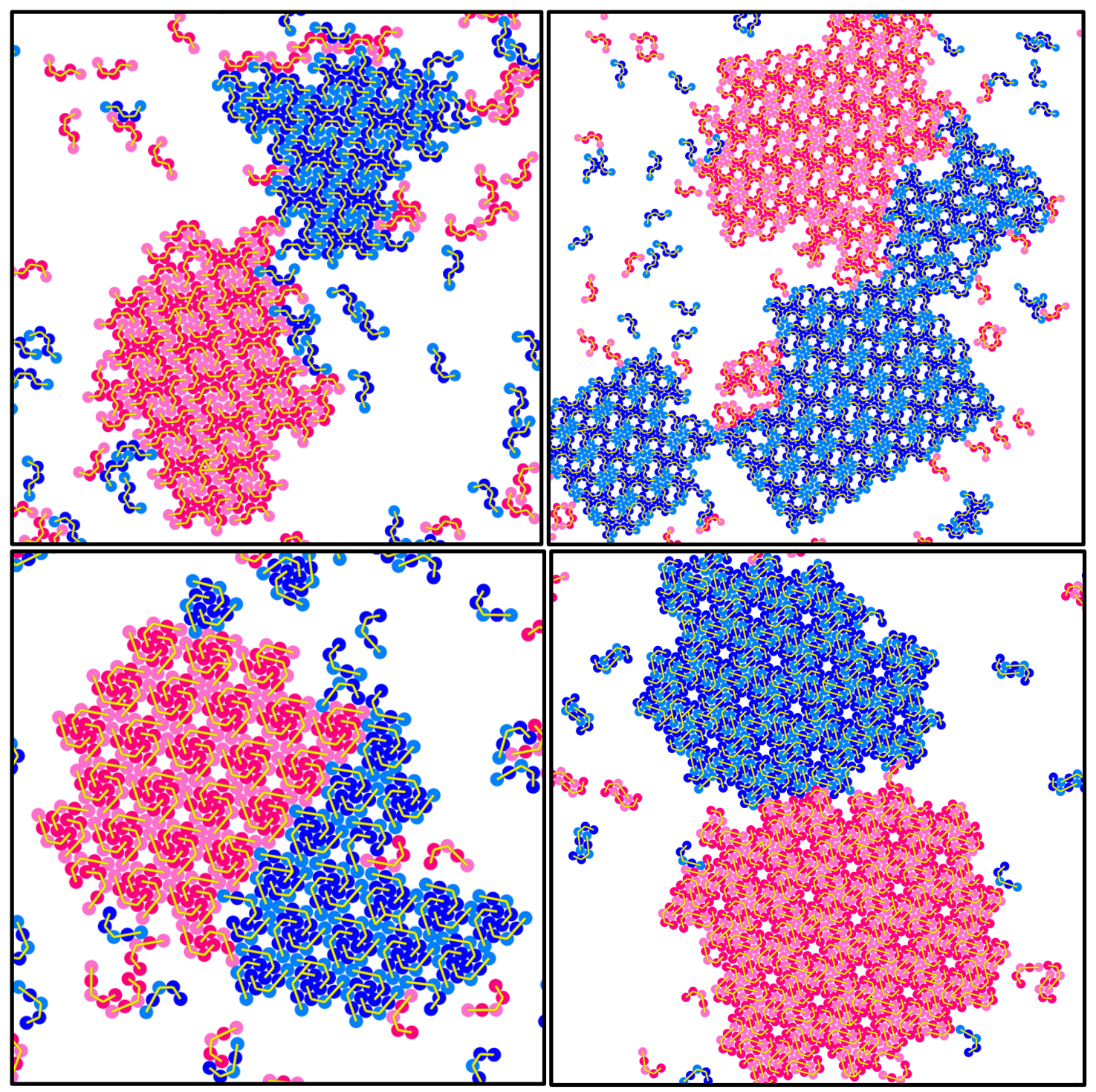

Figure S20: Snapshots of MD simulations of four models with a strong thermodynamic preference for enantiopure crystallization $\left(\Delta E / E_{0} \leq-0.1\right)$, as predicted by the NN. 
6.3. Clustering of Interaction Vectors. In order to determine common features of interaction vectors that produce large positive or large negative $\Delta \mathrm{E}$, corresponding to strong thermodynamic bias for either racemic or enantiopure crystals, respectively, we performed a high-dimensional clustering study on a set of 2000 interaction vectors. These models were obtained by first randomly generating 1,000,000 interaction vectors for a given molecular shape using the Bayesian Bootstrap method as described above, and then selecting the 1000 models with largest and smallest $\Delta \mathrm{E}$, respectively. Clustering of these interaction vectors was performed using $\mathrm{K}$ means and clusters were visualized using t-SNE as implemented in HyperTools. ${ }^{8}$

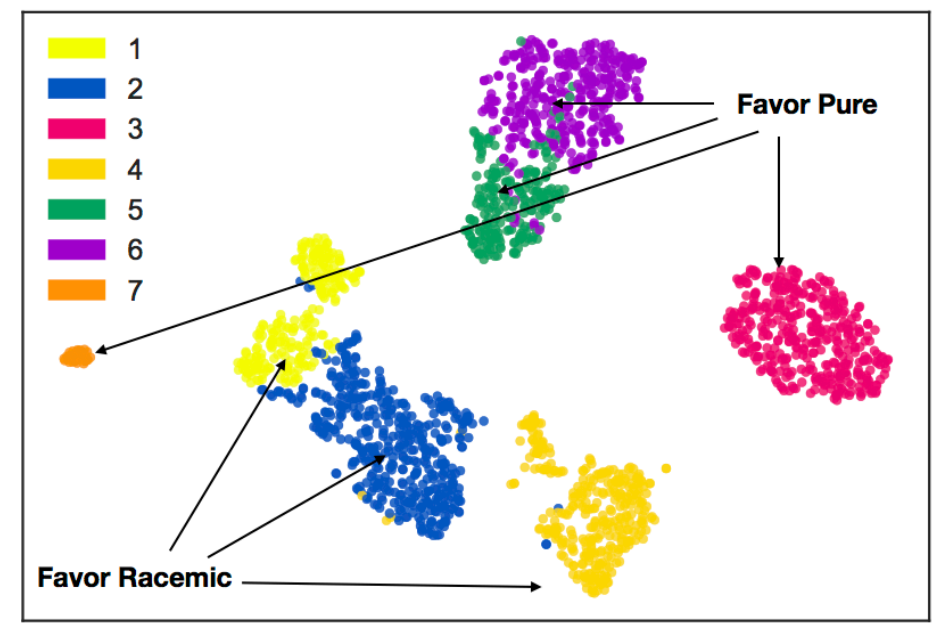

Figure S21: Clustering of 2000 interaction vectors for shape s2 into 7 clusters.

Fig. S21 shows a visualization of such a clustering for molecular shape s2. As evident from the figure, clusters of models that favor racemic crystals are well separated from those that favor enantiopure crystals. This separation directly corresponds to the separation of interaction vectors on the simplex discussed in the main manuscript. For each of the seven clusters, we show the distribution of components of interaction vectors within the cluster in Figure S22. Consistent with the dependence of crystallization propensity on the magnitude of strong interactions $\epsilon_{S}$, as demonstrated in the main manuscript, we find that interaction vectors selected for their large or small $\Delta \mathrm{E}$ are heterogenous: they typically have only one or two strong interactions, resulting in a large $\varphi_{\vec{c}_{p}}$ as defined in the main manuscript. Models with $\epsilon_{S}=5 \epsilon$ have $\varphi_{\vec{c}_{p}}=38^{\circ}$; clusters shown in Figure S22 are even more heterogenous than that (i.e., have larger $\varphi_{\vec{c}_{p}}$ ), as gauged by the average interaction vector of each cluster. This result further supports our observation that heterogenous interactions promote the energetic disparity between different polymorphs and therefore increase crystallization likelihood. 

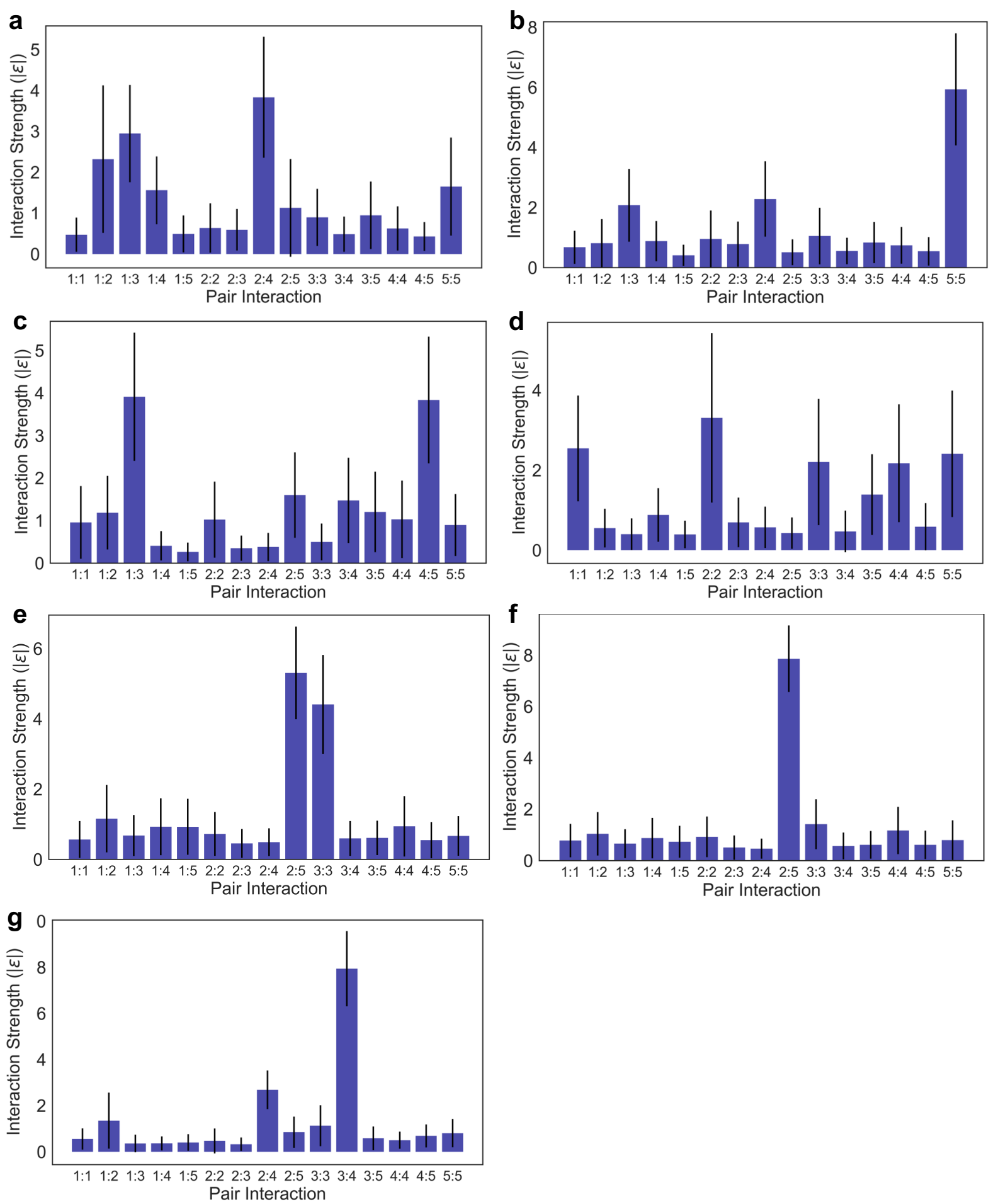

Figure S22: Distribution of the components of interaction vectors in the 7 clusters shown in Fig. S21. Blue bars indicate mean values, black lines represent the standard error of the mean at one standard deviation. (a) cluster $1 ; \Delta E=3.42 \epsilon ; \varphi_{\vec{c}_{p}}=38.1^{\circ}$ (b) cluster 2; $\Delta E=3.45 \epsilon ; \varphi_{\vec{c}_{p}}=46.8^{\circ}$ (c) cluster 3; $\Delta E=-3.65 \epsilon ; \varphi_{\vec{c}_{p}}=40.9^{\circ}$ (d) cluster $4 ; \Delta E=3.37 \epsilon$; $\varphi_{\vec{c}_{p}}=36.9^{\circ}(\mathrm{e})$ cluster $5 ; \Delta E=-3.83 \epsilon ; \varphi_{\vec{c}_{p}}=48.4^{\circ}$ (f) cluster $6 ; \Delta E=-3.87 \epsilon ; \varphi_{\vec{c}_{p}}=54.6^{\circ}$ (g) cluster $7 ; \Delta E=-3.69 \epsilon ; \varphi_{\vec{c}_{p}}=55.9^{\circ}$. 
6.4. Training a $N N$ to Predict $\Delta E$ Based on Descriptors of Molecular Shape. After successfully training a $\mathrm{NN}$ to predict $\Delta \mathrm{E}$ for a specific molecular shape given a random $\vec{\epsilon}$, we attempted to train a NN to predict $\Delta \mathrm{E}$ for molecules of variable interactions and shape. To accomplish this, we added to the input a set of features that encode molecular shape, including the set of 10 distances between pairs of beads in each molecule and the set of 30 angles spanned by triplets of beads. These features were used in addition to the 15 components of the interaction vector to create a 55-dimensional input. In Figure S23a, we evaluate predictions of a NN trained on a data set of $\sim 100,000$ interaction vectors and $\Delta \mathrm{E}$ values for each of the 11 . While the $\mathrm{NN}$ is less accurate than in the case of a fixed molecular shape, reasonable predictive power is achieved.
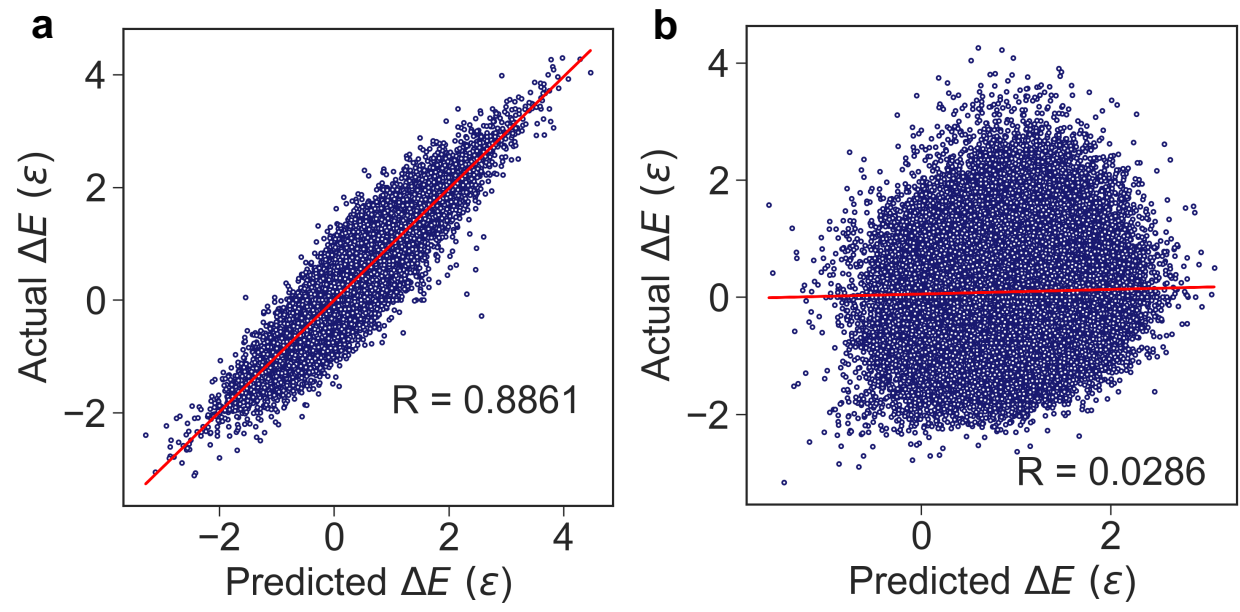

Figure S23: (a) Predicted vs. actual $\Delta \mathrm{E}$ of 100,000 random interaction vectors, using a $\mathrm{NN}$ trained on data including all 11 chiral molecular shapes. (b) Predicted vs. actual $\Delta \mathrm{E}$ of 100,000 random interaction vectors for shape $s 8$, using a NN trained on data including all molecular shapes except $\mathrm{s} 8$. Red lines are best fits to the data.

However, when a single molecular shape is entirely removed from the training set, NN predictions for that specific shape become entirely unreliable, as illustrated in Fig. S23b. This result suggests that the NN is not able to interpolate successfully between different molecular shapes based on the limited number of distinct shapes represented in the training data. This observation is consistent with our inability to deduce shape-specific crystallization tendencies from our molecular dynamics simulations. A much larger set of shapes is likely necessary to analyze the complex interplay of molecular shape, interactions, and crystallization behavior. 


\section{References}

(1) Knuth, D. E. Dancing Links. Millenn. Perspect. Comput. Sci. Proc. 1999 Oxford-Microsoft Symp. Honour Sir Tony Hoare 2000, 187-214.

(2) Booth, K. S. Lexicographically Least Circular Substrings. Inf. Process. Lett. 1980, 10 (45), 240-242.

(3) Damasceno, P. F.; Engel, M.; Glotzer, S. C. Predictive Self-Assembly of Polyhedra into Complex Structures. Science (80-. ). 2012, 337 (6093), 453-457.

(4) Frenkel, D.; Smit, B. Free Energies of Solids. In Understanding Molecular Simulation; Frenkel, D., Smit, B., Eds.; Academic Press: San Diego, 2007; pp 241-267.

(5) Ball, G. H.; Hall, D. J. ISODATA, a Novel Method of Data Analysis and Pattern Classification; 1965.

(6) Rubin, D. B. The Bayesian Bootstrap. Ann. Stat. 1981, 9 (1), 130-134.

(7) Nicholls, J.; Alexander, G. P.; Quigley, D. Polyomino Models of Surface Supramolecular Assembly: Design Constraints and Structural Selectivity. 2017.

(8) Heusser, A. C.; Ziman, K.; Owen, L. L. W.; Manning, J. R. HyperTools: A Python Toolbox for Visualizing and Manipulating High-Dimensional Data. 2017, 1-22. 